\title{
Titel/Title: Elterliche Arbeitslosigkeitsdynamiken und Bildungsverläufe vom Ende der Grundschulzeit bis zum jungen Erwachsenenalter
}

Autor*innen/Author(s): Olaf Groh-Samberg

Henning Lohmann

Veröffentlichungsversion/Published version: Postprint

Zeitschriftenartikel/Journal article

\section{Empfohlene Zitierung/Recommended citation:}

Groh-Samberg, Olaf/ Lohmann, Henning (2017): Elterliche Arbeitslosigkeitsdynamiken und Bildungsverläufe vom Ende der Grundschulzeit bis zum jungen Erwachsenenalter. In:

Kölner Zeitschrift für Soziologie und Sozialpsychologie, 69, 623-650.

Verfügbar unter/Available at:

(wenn vorhanden, bitte den DOI angeben/please provide the DOI if available)

This is a post-peer-review, pre-copyedit version of an article published in Kölner Zeitschrift für Soziologie und Sozialpsychologie. The final authenticated version is available online at: https://doi.org/10.1007/s11577-017-0487-5

Zusätzliche Informationen/Additional information:

Der Autor kann kontaktiert werden unter:

olaf.grohsamberg@uni-bremen.de 
Elterliche Arbeitslosigkeitsdynamiken und Bildungsverläufe vom Ende der Grundschulzeit bis zum jungen Erwachsenenalter

\author{
Henning Lohmann \\ Fachbereich Sozialökonomie \\ Wirtschafts- und Sozialwissenschaftliche Fakultät \\ Universität Hamburg \\ Welckerstr. 8, 20354 Hamburg \\ henning.lohmann@wiso.uni-hamburg.de
}

Tel. 040/42838-8494

Olaf Groh-Samberg BIGSSS

Universität Bremen

Mary-Somerville-Str. 9, 28359 Bremen

ogrohsamberg@bigsss.uni-bremen.de

0421/218-66440 


\title{
Zusammenfassung
}

In der vorliegenden Studie untersuchen wir, welchen Einfluss die elterliche Arbeitslosigkeit auf den Bildungserfolg von Kindern in Deutschland hat. Wir betrachten Bildungsverläufe vom Zeitpunkt des Übergangs in die mehrgliedrige Sekundarstufe bis ins junge Erwachsenenalter von 23 Jahren. Wir fokussieren insbesondere auf die Frage, ob Kinder von Arbeitslosen im Vergleich zu anderen Personen seltener ein Hochschulstudium beginnen, nehmen aber auch den Besuch des Gymnasiums zu Beginn und Ende der Sekundarschulzeit als Bildungsergebnisse mit in den Blick. Wir verwenden Daten des Sozio-oekonomischen Panels (SOEP). Dem Problem der sozialen Selektivität des Arbeitslosigkeitsrisikos begegnen wir mit einem umfassenden Kontrollvariablenansatz im Rahmen von random effects Modellen. Zusätzlich schätzen wir fixed effects Modelle, in denen wir die Tatsache nutzen, dass die Daten Angaben zu Geschwistern enthalten. Im Ergebnis zeigt sich, dass sowohl bei umfassender Kontrolle von Statusmerkmalen als auch in den Geschwistermodellen ein signifikanter Einfluss von Arbeitslosigkeit auf den Bildungserfolg zu beobachten ist. Allerdings gilt dies nicht für alle betrachteten Bildungsergebnisse und auch nicht für alle Ausprägungen von Arbeitslosigkeit.

\begin{abstract}
Parental unemployment dynamics and education trajectories from primary school to early adulthood

In the present study, we examine the influence of parental unemployment on the educational outcomes of children in Germany. We consider the educational pathways from the transition to secondary school until early adulthood at age 23. In particular, we focus on the question whether children of unemployed parents are less likely to enroll in tertiary education.In addition, we also look at educational enrolment at the beginning and the end of secondary school. We use data from the Socio-Economic Panel (SOEP). We address the problem of social selectivity into unemployment by means of a comprehensive control variable approach within the framework of random effects models. In addition, we estimate fixed effects models using siblings' information. As a result, it is evident that a significant influence of unemployment on educational outcomes can be observed both with comprehensive control of status characteristics as well as in sibling models. However, this does not apply to all educational outcomes and not to all forms of unemployment.
\end{abstract}




\section{Elterliche Arbeitslosigkeitsdynamiken und Bildungsverläufe vom Ende der Grundschul- zeit bis zum jungen Erwachsenenalter}

\section{Einleitung}

Die Herkunftsabhängigkeit der Bildungsbeteiligung und des Bildungserfolgs in Deutschland ist breit belegt. Gängige Erklärungsansätze gehen dabei davon aus, dass der Einfluss der sozialen Herkunft sowohl auf die herkunftsspezifische Übertragung von kulturellem Kapital wie auch auf unterschiedliche Bildungsentscheidungen zurückzuführen ist, wobei vor allem elterliche Bildung und Status als entscheidend angesehen werden (Boudon 1974; Bourdieu 1983; Becker 2011). Der soziale Status der Herkunftsfamilie wird dabei als im Wesentlichen konstant angenommen. Als weitere wichtige Einflussfaktoren für den Bildungserfolg gelten jedoch auch kritische Lebensereignisse, die mit Veränderungen in den Bedingungen des Aufwachsens der Kinder verbunden sind. Neben Veränderungen in der Familienstruktur durch Trennung oder Tod eines Elternteils sind dies insbesondere einschneidende Arbeitsmarktereignisse wie die Arbeitslosigkeit der Eltern (Kalil 2013).

Wir betrachten elterliche Arbeitslosigkeit als ein dynamisches Merkmal der sozialen Herkunft, das sich im Bildungsverlauf eines Kindes verändert, während andere Merkmale wie beispielweise die Bildung der Eltern mehr oder weniger stabil sind. Die Berücksichtigung von elterlicher Arbeitslosigkeit verspricht somit ein zusätzliches Potenzial für die Erklärung des unterschiedlichen Bildungserfolgs nach sozialer Herkunft, oder allgemeiner, den unterschiedlichen Bedingungen des Aufwachsens. Arbeitslosigkeit tritt dabei nicht zufällig, sondern sozial selektiv auf und steht somit in engem Zusammenhang mit anderen Herkunftsmerkmalen. Daher ist fraglich, ob el- $(\leftarrow$ p. 624) terliche Arbeitslosigkeit einen eigenständigen Einfluss auf den Bildungserfolg aufweist oder vor allem als Ausdruck anderer Herkunftsmerkmale zu interpretieren ist. Wenn Einflüsse des Ereignisses ,Arbeitslosigkeit' vor allem auf andere Aspekte der sozialen Herkunft zurückzuführen sind, die sowohl auf das Auftreten von Arbeitslosigkeit als auch auf den Bildungserfolg wirken, wäre der Erklärungsgewinn gegenüber statischen Erklärungsmodellen eher gering. Für Deutschland liegen zur Frage des Einflusses der Arbeitslosigkeit auf den Bildungserfolg bislang nur wenige Studien vor (Becker und Nietfeld 1999; Müller et al. 2017). International betrachtet ist die Literatur breiter. Die Studien sind jedoch insbesondere in ihrer Antwort auf die Frage nach einem eigenständigen Einfluss von Arbeitslosigkeit uneindeutig. Tendenziell zeigt sich aber, dass nur ein Teil des negativen Effektes der Arbeitslosigkeit auf andere Statusmerkmale der Eltern zurückzuführen ist und somit elterliche Arbeitslosigkeit einen zusätzlichen Einfluss auf den Bildungsverlauf von Kindern aufweist (Ström 2003; Kalil 2013). 
In der vorliegenden Studie untersuchen wir, welchen Einfluss die elterliche Arbeitslosigkeit auf den Bildungserfolg von Kindern in Deutschland hat. Wir betrachten Bildungsverläufe vom Zeitpunkt des so zentralen Übergangs in die mehrgliedrige Sekundarstufe bis ins junge Erwachsenenalter von 23 Jahren, in dem zumeist die allgemeine Schullaufbahn und teilweise auch Ausbildungen abgeschlossen bzw. nachschulische Bildungswege eingeschlagen sind. Wir fokussieren insbesondere auf die Frage, ob Kinder von Arbeitslosen im Vergleich zu anderen Personen seltener ein Hochschulstudium beginnen, nehmen aber auch den Besuch des Gymnasiums zu Beginn und Ende der Sekundarschulzeit als Bildungsergebnis mit in den Blick. Die Fokussierung auf den Hochschulbesuch ist einerseits dadurch begründet, dass im Zuge der Bildungsexpansion der Übergang ins Studium als sozial selektive Schwelle an Bedeutung gewonnen hat (Müller und Pollak 2004). Andererseits ermöglicht die Betrachtung eines später im Bildungsverlauf auftretenden Übergangs zwischen kurz- und längerfristigen Wirkungen von Arbeitslosigkeit zu unterscheiden. Damit verbunden ist die Frage nach institutionell bedingten Pfadabhängigkeiten und inwiefern diese durch Arbeitslosigkeit ausgelöst oder aber auch verändert werden können: Kann eine durch Arbeitslosigkeit beeinflusste frühe Platzierung im mehrgliedrigen Schulsystem nachhaltige Wirkungen auf spätere Bildungsentscheidungen ausüben? Und können einmal eingeschlagene Bildungspfade durch spätere Ereignisse von elterlicher Arbeitslosigkeit noch in einschneidender Weise verändert werden?

In den Analysen verwenden wir die die Haushaltsdaten des Sozio-oekonomischen Panels (SOEP v31.1). Diese bieten nicht nur die Möglichkeit, den Bildungsverlauf von Kindern über längere Zeiträume nachzuzeichnen. Vor allem können wir auf Grundlage der Daten die materiellen und nicht-materiellen Bedingungen des Aufwachsens umfassend abbilden und dabei zwischen mütterlichen und väterlichen Einflussfaktoren unterscheiden. Diese Informationen nutzen wir zum einen zur Berücksichtigung der sozialen Selektivität der Arbeitslosigkeit, zum anderen zur Modellierung von Faktoren, über die Arbeitslosigkeit auf den Bildungserfolg wirkt (z.B. Einkommensverluste), um auf Mechanismen schließen zu können, über die Arbeitslosigkeit und Bildungserfolg miteinander verknüpft sind. Wie in vielen anderen Studien verfolgen wir mit Blick auf die Selektivitätsproblematik zunächst eine umfassende Kontrollvariablenstrategie. In zusätzlichen Modellen nutzen wir aber auch die $(\leftarrow$ p. 625) Tatsache, dass die Daten Angaben zu Geschwistern enthalten und wir über die Verwendung von fixed effects Schätzern die bestehende Selektivität auch auf andere Art und Weise berücksichtigen können. Im Ergebnis zeigt sich, dass sowohl bei umfassender Kontrolle von Statusmerkmalen als auch in den Geschwistermodellen ein signifikanter Einfluss von Arbeitslosigkeit auf den Bildungserfolg zu beobachten ist. Allerdings gilt dies nicht für alle betrachteten Bildungsergebnisse und auch nicht für alle Ausprägungen von Arbeitslosigkeit. Vor allem längere bzw. mehrfach auftretende 
Phasen väterlicher Arbeitslosigkeit haben einen nachhaltig negativen Einfluss auf den Bildungserfolg.

\section{Arbeitslosigkeit, Bildungserfolg und soziale Herkunft}

Arbeitslosigkeit ist ein Ereignis, das negativ auf materielle und immaterielle Merkmale von Familien wirkt. Erwartungsgemäß zeigt sich in den überwiegend für angelsächsische Länder vorliegenden Studien zumindest in bivariaten, häufig auch in multivariaten Analysen ein negativer Zusammenhang zwischen elterlichen Arbeitslosigkeitserfahrungen und dem Bildungserfolg von Kindern (vgl. für einen aktuellen Überblick Kalil 2013). Auch die wenigen Studien, die Deutschland betrachten, kommen zum Ergebnis eines geringeren Bildungserfolgs von Kindern von Arbeitslosen, die seltener das Gymnasium oder Hochschulen besuchen sowie insgesamt kürzere Bildungsdauern aufweisen (Becker und Nietfeld 1999; Müller et al. 2017). Entsprechend formulieren wir folgende Ausgangshypothese: Der Bildungserfolg von Kindern, deren Eltern während ihres Bildungsverlaufs arbeitslos waren, ist geringer als von Kindern, deren Eltern nicht arbeitslos waren (H1).

Nicht eindeutig ist dagegen der Forschungsstand hinsichtlich der Frage, ob der in den vorliegenden Studien beobachtete Zusammenhang kausal auf Arbeitslosigkeit oder auf andere Faktoren zurückzuführen ist, die sowohl auf das Arbeitslosigkeitsrisiko als auch den Bildungserfolg wirken. Gedacht ist hierbei vor allem an elterliche Statusmerkmale wie Bildung oder Beruf. Um auszuschließen, dass der Zusammenhang zwischen Arbeitslosigkeit und Bildungserfolg allein auf die soziale Selektivität des Arbeitslosigkeitsrisikos zurückzuführen ist, verwenden die vorliegenden Studien unterschiedliche Analysedesigns. ${ }^{1}$ Hierzu zählen insbesondere die Verwendung möglichst umfangreicher Kontrollvariablen (Dronkers 1994; Kalil und Ziol-Guest 2008; Kalil und Wightman 2011), die Anwendung von Matching Verfahren (Brand und Thomas 2014; Peter 2016) und fixed effects Schätzern, die entweder die Variation über die Zeit (Levine et al. 2011; Stevens und Schaller 2011) oder zwischen Geschwistern (Ermisch et al. 2004; Levine et al. 2011; Müller et al. 2017) ( $\leftarrow$ p. 626) zur Identifikation kausaler Effekte nutzen. Die meisten Studien stellen auch nach Berücksichtigung möglicher Selektivität einen Zusammenhang zwischen Arbeitslosigkeit und Bildungserfolg fest, wobei dies sowohl für Studien mit einfachen und komplexeren Designs zutrifft. Dagegen findet beispielsweise Dronkers (1994) in Modellen mit Kontrollvariablen keinen signifikanten Einfluss der Arbeitslosigkeit auf die Bildungsbeteiligung, wohl aber einen signifikant negativen auf das Abschneiden in Kompetenztests. Levine

\footnotetext{
${ }^{1}$ Sehr breit wird die Frage des eigenständigen Einflusses kritischer Lebensereignisse in der Literatur zum Einfluss von Trennung und Scheidung auf den Bildungserfolg von Kindern diskutiert (vgl. grundlegendend Manski et al. 1992). Mit Blick auf Arbeitslosigkeit besteht dasselbe Grundproblem, sodass sich Argumentation und empirische Vorgehensweisen ähneln.
} 
et al. (2011) schließen aufgrund der Ergebnisse von fixed effects Modellen das Vorliegen eines kausalen Arbeitslosigkeitseffekts aus. Müller et al. (2017) kommen unter Kontrolle konstanter beobachteter und unbeobachteter familialer Merkmale zu uneindeutigen Ergebnissen. Insgesamt ist der Forschungsstand somit uneinheitlich, wobei die größere Zahl der Studien einen Einfluss der Arbeitslosigkeit auch nach Berücksichtigung der sozialen Selektivität des Arbeitslosigkeitsrisikos feststellen kann. Selbst wenn der Arbeitslosigkeitseffekt nicht vollständig auf andere Merkmale zurückzuführen ist, gehen wir davon aus, dass die soziale Selektivität der Arbeitslosigkeit bedeutsam und somit notwendigerweise in den Analysen zu beachten ist. Entsprechend formulieren wir folgende Hypothese als striktere Version der ersten Hypothese: Auch unter Berücksichtigung der sozialen Selektivität der Arbeitslosigkeit unterscheidet sich der Bildungserfolg von Kindern, deren Eltern während ihres Bildungsverlaufs arbeitslos waren, von Kindern, deren Eltern nicht arbeitslos waren (H2).

Liegt auch bei Berücksichtigung sozialer Selektivität ein Zusammenhang zwischen Arbeitslosigkeit und Bildungserfolg vor, kann dieser auf unterschiedliche Mechanismen zurückzuführen sein. Arbeitslosigkeit wirkt negativ auf eine Reihe von Faktoren, die wiederum negativ auf den Bildungserfolg wirken. Eine Orientierung für die Systematisierung möglicher Mediatoren bietet die allgemeine Unterscheidung zwischen primären und sekundären Effekten der sozialen Herkunft (Boudon 1974). Arbeitslosigkeit ist mit dem Verlust von Erwerbseinkommen verbunden, der nicht (vollständig) durch Transfers oder andere Einkommen ausgeglichen wird. Frühere Studien zeigen, dass Einkommensverluste bei Arbeitslosigkeit zu einem größeren Teil für den negativen Einfluss auf den Bildungserfolg verantwortlich sind (Becker und Nietfeld 1999; Coelli 2011; Kalil und Wrightman 2011; Kalil und Ziol-Guest 2008). Nimmt man direkte und indirekte Kosten der Beschulung (insbesondere Opportunitätskosten) als relevante Parameter der Entscheidung für Bildungswege, die zu höheren Abschlüssen führen, ist Arbeitslosigkeit als Teil des sekundären Effekts der sozialen Herkunft zu interpretieren. Ergänzend, aus unserer Sicht aber weniger relevant, ist die Annahme eines finanziellen Einflusses auf Schulleistungen, der über bezahlte Nachhilfe u.ä. erreicht wird.

Neben ökonomischen Einbußen sind weitere negative Konsequenzen der Arbeitslosigkeit denkbar, die den Bildungserfolg von Kindern beeinflussen können. Arbeitslosigkeit wirkt auf elterliche Partnerschaften, das Familienklima und damit verbunden die Unterstützung von Kindern. Im Sinne primärer Herkunftseffekte ist hier ein negativer Einfluss auf schulische Leistungen aufgrund weniger unterstützender bzw. konflikthafter Familienbeziehungen anzunehmen. Entsprechend berück- $(\leftarrow$ p. 627) sichtigen Studien zum Zusammenhang zwischen Arbeitslosigkeit und Bildungserfolg Faktoren wie familiale Aktivitäten (Dronkers 1994), elterliche Trennungen, Scheidungen und damit verbundene räumliche Mobilität (Levine et al. 2011; Stevens 
und Schaller 2011). ${ }^{2}$ Die vorliegenden Studien zeigen, dass diese Faktoren im Vergleich zu den ökonomischen Faktoren nur einen geringen Einfluss aufweisen, aber auch nicht vollständig vernachlässigbar sind.

Als dritter Faktor sind Auswirkungen der mit der Arbeitslosigkeit verbundenen Statusverluste und Unsicherheiten zu berücksichtigen (Andersen 2013, siehe für eine analoges Argument im Kontext von Scheidungsfolgen Jonsson und Gähler 1997), die entsprechend geringere Bildungsaspirationen zur Folge haben könnten. Es liegen bislang keine Studien vor, welche die Auswirkungen der über die direkten ökonomischen Folgen von Arbeitslosigkeit hinausreichenden ökonomischen Unsicherheit gezielt untersuchen. Lauterbach und Lange (1998) zeigen jedoch in einer Studie zum Einfluss von Armut auf den Bildungserfolg von Kindern, dass wirtschaftliche Sorgen einen Einfluss über die reine Armutslage hinaus aufweisen. Wir erwarten, dass hierüber auch ein Teil des negativen Einflusses elterlicher Arbeitslosigkeit auf den Bildungserfolg zu erklären ist.

Insgesamt gehen wir allgemein von einer Mediationshypothese aus: Der Einfluss elterlicher Arbeitslosigkeit auf den Bildungserfolg wird über unterschiedliche familiale Faktoren vermittelt, auf die Arbeitslosigkeit einen Einfluss aufweist. Aufgrund des Forschungsstandes erscheinen uns hierbei drei Faktoren zentral, zu denen wir folgende Hypothesen formulieren: Elterliche Arbeitslosigkeit wirkt negativ auf das Einkommen, die familialen Beziehungen und die wahrgenommene ökonomische Unsicherheit: je niedriger das elterliche Einkommen, desto niedriger die Wahrscheinlichkeit des Bildungserfolgs (H3a); je weniger unterstützend die familialen Beziehungen, desto niedriger die Wahrscheinlichkeit des Bildungserfolgs (H3b); je höher die wahrgenommene ökonomische Unsicherheit (Sorgen), desto niedriger die Wahrscheinlichkeit des Bildungserfolgs ( $\mathrm{H} 3 \mathrm{c})$.

Der formale Bildungsverlauf, begonnen mit der Einschulung bis zum Abschluss von Berufsausbildungen oder Studiengängen, umfasst einen langen Zeitraum, in dem Arbeitslosigkeit von Eltern auftreten kann. Aufgrund der geringen Durchlässigkeit des mehrgliedrigen Bildungssystems ist die frühere Platzierung im Bildungssystem entscheidend für spätere Übergänge (Maaz et al. 2009; Becker 2011). Wirkt Arbeitslosigkeit negativ auf die frühe Platzierung, kann diese darüber vermittelt auch auf $(\leftarrow$ p. 628) spätere Bildungsergebnisse wirken. Die

\footnotetext{
2 Im Gegensatz dazu lässt sich argumentieren, dass im Fall von Arbeitslosigkeit mehr Zeit mit den Kindern verbracht wird und dies den Bildungserfolg positiv beeinflusst. Levine et al. (2011) finden aber keine deutlichen empirischen Belege für diese Hypothese - was den seit der Marienthal-Studie bekannten Befunden der Arbeitslosigkeitsforschung entspricht, dass das Mehr an verfügbarer Zeit häufig nicht in gewinnbringende Aktivitäten umgesetzt werden kann (vgl. Rogge 2013).
} 
entsprechende Hypothese kann als Unterhypothese der Mediationshypothese betrachtet werden, wobei wir nun allerdings auf institutionelle und nicht auf familiale Faktoren fokussieren. Da wir in den folgenden Analysen umfassend auf den Einfluss der früheren Platzierung im Bildungssystem eingehen, heben wir sie als ,Pfadabhängigkeitshypothese' hervor: Eine Platzierung in niedrigeren Bildungsgängen im früheren Bildungsverlauf wirkt negativ auf den späteren Bildungserfolg $(\mathrm{H} 3 \mathrm{~d})$.

Arbeitslosigkeitserfahrungen können sich nach Zeitpunkt und Dauer unterscheiden. Außerdem können im Fall elterlicher Arbeitslosigkeit der Vater oder die Mutter betroffen sein. Wenn die konkrete Ausprägung von Arbeitslosigkeit für den Bildungserfolg bedeutsam sein sollte, ist es notwendig, die erste allgemeine Hypothese durch präziser formulierte Hypothesen zu ergänzen. In Hinblick auf den Bildungsverlauf stellt sich die Frage, welchen Einfluss der Zeitpunkt des Auftretens von Arbeitslosigkeit für den späteren Bildungserfolg hat. Eine nachhaltige Wirkung von elterlicher Arbeitslosigkeit sollte sich vor allem dann ergeben, wenn sie sich negativ auf Übergangsentscheidungen im Bildungsverlauf auswirkt. Die empirische Evidenz zu dieser Frage ist spärlich und uneindeutig. Während Ermisch et al. (2004) sowohl einen Einfluss früher und später Arbeitslosigkeitsepisoden feststellen (aber nicht dazwischenliegender), zeigt sich in der Studie von Brand und Thomas (2014) allein ein Einfluss nahe am Bildungsübergang liegender Arbeitslosigkeitserfahrungen. Auch wir gehen von einem stärkeren Einfluss der nahe an Bildungsübergängen liegenden Arbeitslosigkeitserfahrungen aus: Je zeitlich näher die elterliche Arbeitslosigkeitserfahrung an einem Bildungsübergang liegt, desto stärker negativ wirkt sie auf den Erfolg bei diesem Bildungsübergang ( $\mathrm{H} 4 \mathrm{a})$. Weiter zeigen vorliegende Studien, dass auch die Dauer der Arbeitslosigkeit einen Einfluss auf den Bildungserfolg aufweist. Je länger die Arbeitslosigkeit ist, desto niedriger der Bildungserfolg (Becker und Nietfeld 1999; Kalil und Wightman 2008; Levine et al. 2008). Zu vermuten ist, dass die im Zusammenhang mit der dritten Hypothese diskutierten Faktoren bei längerer Arbeitslosigkeit in verstärktem Maße auftreten. Entsprechend und im Einklang mit früherer Forschung formulieren wir die $\mathrm{Hy}-$ pothese: Je anhaltender die elterliche Arbeitslosigkeitserfahrung ist, desto stärker negativ wirkt sie auf den Bildungserfolg ( $\mathrm{H} 4 \mathrm{~b})$. Einige Studien unterscheiden zwischen mütterlicher und väterlicher Arbeitslosigkeit bzw. zwischen der Arbeitslosigkeit von Haupt- und Nebenverdiener/innen. Diese zeigen, dass die mütterliche Arbeitslosigkeit bzw. die Arbeitslosigkeit von Nebenverdiener/-innen keinen oder nur einen schwachen Einfluss auf den Bildungserfolg von Kindern aufweist, was vor allem über die geringeren Einkommensverluste $(\leftarrow$ p. 629) und darüber zu erklären ist, dass die Arbeitslosigkeit von Vätern aufgrund sozialer Normen familial belastender ist. Wir gehen daher von folgender Hypothese aus: Die väterliche Arbeitslosigkeit wirkt stärker negativ auf den Bildungserfolg als die mütterliche Arbeitslosigkeit $(\mathrm{H} 4 \mathrm{c})$. 
Die Überprüfung der ersten drei Hypothesen(bündel) erfolgt entlang von zwei zentralen Fragen für die folgenden empirischen Analysen. Weist elterliche Arbeitslosigkeit einen eigenständigen, über andere Statusmerkmale hinausgehenden Einfluss auf den Bildungserfolg auf $(\mathrm{H} 1$ und H2)? Und falls ja, über welche Mechanismen wirkt die Arbeitslosigkeit auf den Bildungsverlauf $(\mathrm{H} 3)$. Hierbei unterscheiden wir zwischen familialen Mediatoren und institutionellen Abhängigkeiten. Das vierte Hypothesenbündel $(\mathrm{H} 4)$ präzisiert die vorangestellten Hypothesen hinsichtlich der Art (Zeitpunkt, Dauer, Geschlechtsspezifik) der Arbeitslosigkeit.

\section{Datenbasis und methodisches Vorgehen}

Für die empirische Überprüfung der Hypothesen nutzen wir Daten des Sozio-oekonomischen Panels (SOEP), einer seit 1984 jährlich durchgeführten Haushaltsbefragung (Wagner et al. 2007). Befragt werden alle Personen in den Befragungshaushalten ab 17 Jahren. Angaben zu Kindern bis 16 Jahren, darunter auch Angaben zur Bildungsbeteiligung, werden über einen gesonderten Haushaltsfragebogen erhoben. Personen, die einen Haushalt verlassen, werden in ihrem neuen Haushalt weiterbefragt. Daher ist es möglich, Bildungsverläufe von Kindern und jungen Erwachsenen über einen längeren Zeitraum im Kontext des elterlichen Haushalts und auch nach dem Verlassen dieses Haushalts zu analysieren (Lohmann et al. 2009).

Aufgrund der zentralen Bedeutung der Platzierung im mehrgliederigen Schulsystem nach Ende der Grundschulzeit wählen wir als Ausgangspunkt für unser Beobachtungsfenster den Zeitpunkt des Übergangs in die Sekundarstufe, der in der Regel im Alter von zehn bis zwölf Jahren erfolgt. Auf Basis von Retrospektivinformationen berücksichtigen wir aber auch das Auftreten elterlicher Arbeitslosigkeit, die vor diesem Zeitpunkt liegt. Uns interessiert dabei, ob frühe Phasen der Arbeitslosigkeit langfristig auf den Bildungsverlauf der Kinder einwirken und ob spätere Arbeitslosigkeitsphasen dazu führen, dass - trotz einer immer noch recht geringen Mobilität zwischen Bildungsgängen in Deutschland - Abweichungen von einem bereits eingeschlagenen Bildungsweg erfolgen. Entsprechend dieser Überlegungen reicht unser Analysefenster bis zum Alter von 23 Jahren, bis zu dem für einen größeren Teil der Population eine erste Entscheidung über nachschulische Bildungswege erfolgt ist.

Aufgrund des weit zurückreichenden Analysezeitraums und der unterschiedlichen Entwicklung der Arbeitslosigkeit in West- und Ostdeutschland betrachten wir ausschließlich Westdeutschland. Da wir die Wirkungen mütterlicher und väterlicher Arbeitslosigkeit untersuchen, beschränken wir die Analysen auf Kinder, deren Eltern bis mindestens zum Ende der Sekundarschulzeit zusammengelebt haben. Den Vater und die Mutter eines Kindes identifizieren wir über die im SOEP verfügbaren Zeiger, die auf zwei unterschiedliche Informationen zugreifen. 
Dies sind die Anga- $(\leftarrow$ p. 630$)$ ben aus der einmalig erhobenen Geburtenbiographie aller Befragungspersonen und die Angaben zur Stellung zum ,Haushaltsvorstand', die jährlich mit der Haushaltsbefragung erhoben werden. Wir definieren Mutterschaft und Vaterschaft als konstantes Merkmal, berücksichtigen also keine Personen, die im Zuge neuer Familienkonstellationen die Vater- oder Mutterrolle übernehmen. Die Fallzahl für diesen mehr als zehn Beobachtungsjahre umfassenden Längsschnitt beträgt $n=963$. Zusätzliche Analysen für Ostdeutschland und für Alleinerziehende wären wünschenswert, lassen sich aber aufgrund der geringen Fallzahlen nicht realisieren. Analysen, in denen wir Gesamtdeutschland bzw. alle Familien berücksichtigen (Ergebnisse nicht berichtet), bieten aber keinen Anlass dafür anzunehmen, dass sich die grundlegenden Muster in Ostdeutschland oder für Alleinerziehende gegenüber westdeutschen Paarhaushalten stark unterscheiden.

In den zentralen Analysen betrachten wir als abhängige Variable die Aufnahme eines Hochschulstudiums. Außerdem betrachten wir in zusätzlichen Analysen frühere Ereignisse im Bildungsverlauf: den Besuch des Gymnasiums nach Ende der Grundschulzeit und beim Verlassen der Schule. Bei der Erhebung von Schulabschlüssen von Schulabgängern im SOEP tritt ein höherer Anteil von fehlenden Werten auf, sodass nicht immer eindeutig zuzuordnen ist, ob ein Schulabschluss nicht erworben oder nicht erhoben wurde (Lohmann und Witzke 2011, S.22). Da dies zu Verzerrungen führt, greifen wir auf die Angaben zum besuchten Schultyp zum Ende der Schulzeit zurück.

Tabelle A1 im Anhang bietet einen Überblick über Mittelwert, Standardabweichung, Minimum und Maximum aller verwendeten Variablen. Einige der Variablen wie beispielsweise das Haushaltseinkommen weisen einen höheren Anteil fehlender Werte auf. Beim Einkommen verwenden wir die imputierten Jahreseinkommensangaben, wie sie standardmäßig im SOEP vorliegen (Grabka 2016). Insbesondere Angaben zum sozio-ökonomischen Status, aber auch zur elterlichen Bildung liegen nicht immer für alle Beobachtungsjahre vor. Hier greifen wir vorwiegend auf zurückliegende Jahre zurück, in denen diese Angaben vorliegen. Ein geringer Anteil der Angaben zum sozio-ökonomischen Status (etwa 1 Prozent) wurde über eine regressionsbasierte Imputation ersetzt. Weiter liegen nicht immer vollständige Angaben zur Erwerbsbiographie der Eltern und somit zu Arbeitslosigkeitsphasen vor. Dies ist aber vor allem der Fall, wenn Kinder nicht dauerhaft mit beiden Elternteilen zusammenleben. In der für die Analysen zentralen Stichprobe kommt dies nur in wenigen Ausnahmenfällen vor. Trotzdem wurden diese fehlenden Informationen unter Verwendung der vorliegenden Angaben zur Erwerbstätigkeit, zur Bildung, zum Alter, zum Haushaltstyp und zum Bundesland imputiert. Nach Durchführung dieser Schritte weisen weniger als zehn der Beobachtungen fehlende Werte in mindestens einer der übrigen Variablen auf. Diese Beobachtungen wurden von den Analysen 
ausgeschlossen, was aufgrund des geringen Umfangs keinen substantiellen Einfluss auf die Ergebnisse haben sollte.

Wir präsentieren die Ergebnisse von linearen Wahrscheinlichkeitsmodellen, die wir aufgrund der einfacheren Schätzbarkeit und Interpretierbarkeit Logit-Modellen vorziehen. Die Ergebnisse von zusätzlich geschätzten Logit-Modellen unterscheiden $(\leftarrow$ p. 631) sich nur geringfügig von den im Folgenden dargestellten Ergebnissen (siehe auch Abschnitt 5). Im Datensatz sind teilweise mehrere Geschwister aus einer Familie enthalten, wobei wir die Geschwisterbeziehungen über die gemeinsame Mutter identifizieren. Die 963 Kinder im Datensatz stammen von 701 Müttern. Wir berücksichtigen die aus dem Vorhandensein von Geschwistern im Datensatz resultierende Verletzung der Annahme der Unabhängigkeit der Beobachtungen über eine random effects (RE) Modellierung. In weiteren Analysen nutzen wir die Abhängigkeit der Beobachtungen, um für unbeobachtete Herkunftseffekte zu kontrollieren. Wir verwenden fixed effects Schätzer, die allein die Varianz zwischen Geschwistern nutzen. Dies erlaubt, die für Regressionsmodelle grundlegende Exogenitätsannahme weniger strikt zu formulieren. Es liegt auch dann keine Annahmeverletzung vor, wenn relevante konstante Familienmerkmale unbeobachtet bleiben. Entsprechend sollten fixed effects Schätzer im Vergleich zu herkömmlichen oder random effects Regressionsschätzern eher geeignet sein, Aussagen über kausale Zusammenhänge empirisch abzusichern. Somit können wir über dieses Vorgehen vollständig ausschließen, dass der beobachtete Einfluss der elterlichen Arbeitslosigkeit auf konstante (Status-)Merkmale der Eltern zurückzuführen ist. Dies betrifft den zentralen Punkt der von uns formulierten Selektivitätshypothese, dem wir bislang über die möglichst umfassende Kontrolle beobachtbarer Merkmale begegnet sind.

Die fixed-effects Schätzer können aber weiterhin aufgrund unbeobachteter, aber zeitlich variabler Familienmerkmale oder von unbeobachteten Eigenschaften der Kinder verzerrt sein. Ein spezifisches Problem besteht in der geringen Effizienz der fixed-effects Schätzung. Der Einfluss der Arbeitslosigkeit auf den Bildungserfolg wird allein über die Varianz zwischen Geschwistern identifiziert. Beginnt ein erstes Kind einer Familie kein Studium, aber das zweite und ist der Vater während der Sekundarschulzeit des ersten Kindes arbeitslos, aber nicht während der eines zweiten Kindes, wird ein negativer Einfluss der Arbeitslosigkeit identifiziert. Da die Varianz zwischen Geschwistern geringer ist als zwischen Familien, aber nur erstere genutzt wird, ist die Schätzung im Vergleich zu anderen Regressionsmodellen ineffizient. Ein drittes Problem besteht in der Selektivität der Stichprobe. Diese enthält keine Einzelkinder und auch keine Personen mit Geschwistern, deren Bildungsverläufe nicht in den Beobachtungszeitraum fallen. Wir berichten daher die Ergebnisse nur zusätzlich zu den vorherigen Analysen. 


\section{Variablen und Modelle}

Um kurz- und längerfristige Einflüsse elterlicher Arbeitslosigkeit in den Blick nehmen zu können, betrachten wir als Indikator des Bildungserfolgs die Wahrscheinlichkeit des Studienbeginns. In der Darstellung der Ergebnisse der empirischen Analysen orientieren wir uns an den vorherigen theoretischen Überlegungen und daraus abgeleiteten Hypothesen. Zuerst betrachten wir ohne Berücksichtigung weiterer Variablen den Zusammenhang zwischen Arbeitslosigkeit und Studienwahrscheinlichkeit. Danach gehen wir in weiteren Modellen auf die Fragen der sozialen Selektivität, der Mediation des Arbeitslosigkeitseinflusses über weitere Variablen und die Bedeutung des Bildungsverlaufs ein. Insgesamt stellen wir die Ergebnisse von sechs $(\leftarrow$ p. 632) unterschiedlichen, stufenweise aufgebauten Modellen dar. Da wir diese Form der Modellierung auch im weiteren Verlauf der Analysen mit zusätzlichen abhängigen Variablen verwenden, wir aber nicht immer die vollständigen Modelltabellen darstellen, erläutern wir den Aufbau der Modelle etwas ausführlicher. Dabei gehen wir auch auf die konkrete Operationalisierung der Variablen ein. Tabelle 1 enthält eine Übersicht über die in den einzelnen Modellen enthaltenen Variablen.

Modell 1 umfasst die für die Ausgangshypothese relevanten Arbeitslosigkeitsvariablen. Wir betrachten dabei zwei sechs bzw. sieben Jahre umfassende Phasen, in denen Arbeitslosigkeit auftreten kann. Die erste Phase umfasst die vollständige Grundschulzeit (Alter 5 bis 10 Jahre), die zweite fällt in die Sekundarschulzeit (11 bis 17 Jahre). Zur Bestimmung von Arbeitslosigkeit verwenden wir die Erwerbskalendarien des SOEP auf jährlicher Basis. Jede der Variablen hat drei Ausprägungen: keine Arbeitslosigkeit in der jeweiligen Phase, Arbeitslosigkeit in einem Jahr und Arbeitslosigkeit in zwei oder mehr Jahren. ${ }^{3}$ Alle dargestellten Modelle enthalten gleichzeitig die Arbeitslosigkeitsvariablen der Mutter und des Vaters. Die Ergebnisse sind also als Einfluss der Arbeitslosigkeit unter Kontrolle der Arbeitslosigkeit des jeweiligen anderen Elternteils zu interpretieren. In zusätzlichen Modellen, in denen wir jeweils nur die Arbeitslosigkeitsvariablen der Mutter bzw. des Vaters berücksichtigen, zeigen sich keine grundsätzlich anderen Ergebnisse (nicht dargestellt).

Modell 2 enthält eine Reihe von Merkmalen des Kindes, der Eltern und des Haushalts, die wir als Kontrollvariablen ansehen. Dies sind: Geschlecht und Migrationshintergrund des Kindes,

\footnotetext{
${ }^{3}$ Aufgrund der Nutzung der Daten des jährlichen Erwerbskalendariums, ist es nicht möglich, die Dauer des Auftretens von Arbeitslosigkeit zu berücksichtigen, da jeweils nur die Information vorliegt, ob eine Person in einem Jahr arbeitslos war oder nicht. In zusätzlichen Analysen haben wir die Angaben des monatlichen Erwerbskalendariums verwendet, die allerdings nur für einen kürzeren Abschnitt des Bildungsverlaufs vorliegen. Diese Analysen zeigen (Ergebnisse nicht dargestellt), dass Arbeitslosigkeitsdauern von mehr als 6 Monaten negativ mit dem Bildungserfolg korreliert sind, während dies für kürzere Arbeitslosigkeitsdauern nicht gilt.
} 
Alter der Mutter bei Geburt, Haushaltsgröße, das Bundesland und den Zeitpunkt des Übergangs in die Sekundarstufe. Um mögliche Effekte einer aus einem neuen Job nach Arbeitslosigkeit resultierenden räumlichen Mobilität abzubilden, verwenden wir zwei weitere Kontrollvariablen. Die erste verweist auf Umzüge seit Beginn der Schulzeit bis zum Übergang in die Sekundarstufe, die zweite auf Umzüge vom Übergang in die Sekundarstufe bis zum Ende der Schulzeit. Die Grundlage für diese Variablen bilden Informationen zum Einzugsjahr in der gegenwärtigen Wohnung, die sowohl retrospektiv als auch prospektiv erhoben werden.

Modell 3 fügt zentrale Statusmerkmale hinzu und zielt auf die Frage, inwieweit in vorherigen Modellen beobachtete Einflüsse der elterlichen Arbeitslosigkeit auf die beobachtbare soziale Selektivität des Arbeitslosigkeitsrisikos zurückzuführen sind. Konkret enthält das Modell eine Variable zum höchsten elterlichen Bildungsabschluss und zum sozio-ökonomischen Status (ISEI), wobei auch hier jeweils der höhere Wert von beiden Elternteilen verwendet wird. Aufgrund der geringen Veränderung der Bildung der Eltern im Längsschnitt messen wir diese nur zum Zeitpunkt des Übergangs in die Sekundarstufe. Da sich der sozio-ökonomische Status verändern kann, u.a. aufgrund von arbeitslosigkeitsbedingten Jobwechseln, berechnen wir $(\leftarrow$ p. 633) den Durchschnittswert des ISEI vom Zeitpunkt des Übergangs in die Sekundarstufe bis zum Ende der Schulzeit.

Modell 4 und 5 enthalten Variablen, über die mögliche vermittelnde Faktoren berücksichtigt werden. Dies sind die relative Einkommensposition des Haushalts als Lebensstandardmaß und Angaben zu wirtschaftlichen Sorgen als Indikator für die subjektive Wahrnehmung ökonomischer Unsicherheit. Die relative Einkommensposition operationalisieren wir als bedarfsgewichtetes Haushaltsnettoeinkommen im Verhältnis zum jeweiligen Einkommensmedian in der Gesamtbevölkerung (arithmetisches Mittel über alle Jahre der Sekundarschulzeit). Die wirtschaftlichen Sorgen messen wir jeweils über den höheren Wert von Mutter oder Vater.

Angaben zu familialen Beziehungen liegen für das Gesamtsample nicht vor, werden aber in zusätzlichen Analysen verwendet. Hierfür nutzen wir Angaben aus der Befragung über den sogenannten Jugendfragebogen des SOEP, in dem seit dem Jahr 2000 17-jährige zu verschiedenen Aspekten des Aufwachsens befragt werden. Die Fragen beziehen sich entweder auf den Zeitpunkt der Befragung oder sind retrospektiv angelegt. Es handelt sich dabei nicht um zeitveränderliche Angaben und sie stehen auch nur für einen kleinen Teil der Gesamtstichprobe zur Verfügung. Um trotzdem eine ausreichend große Fallzahl zu erzielen ( $n=900)$, verzichten wir in den Analysen auf Angaben, die sich auf den Zeitpunkt des Übergangs in die Sekundarstufe beziehen. Diese Stichprobe verwenden wir für zusätzliche Analysen, um mög- 
liche Zusammenhänge zwischen elterlicher Arbeitslosigkeit, Erziehungsstilen und Schulleistungen mitberücksichtigen zu können. Erziehungsstile werden im Jugendfragebogen über die deutsche Version der „Supportive Parenting Scale“ (vgl. Schwarz und Walper 1997) mit neun Items abgebildet, die wir in einem ungewichteten additiven Index für die Mutter und den Vater zusammenführen. ${ }^{4}$ Als Indikator für Schulleistungen verwenden wir die Mathematiknote mit 17 Jahren bzw. bei Abschluss der Schule und die Information, ob bis zu diesem Zeitpunkt eine Klasse wiederholt wurde.

\section{Elterliche Arbeitslosigkeit und Wahrscheinlichkeit des Studienbeginns}

Abbildung 1 fasst die Ergebnisse zum Zusammenhang zwischen der Arbeitslosigkeit des Vaters und der Wahrscheinlichkeit ein Studium zu beginnen zusammen. Dargestellt sind jeweils der Koeffizient und das jeweilige 95\%-Konfidenzintervall für alle sechs in Tabelle 1 erläuterten Modelle. Die Koeffizienten und Standardfehler aller Variablen sind in Tabelle A2 im Anhang dargestellt. ${ }^{5}$ Die Modellergebnisse zu wei- $(\leftarrow$ p. 634) teren Faktoren neben der Arbeitslosigkeit bestätigen den breiten Forschungsstand zur Herkunftsabhängigkeit von Bildungsergebnissen, weswegen wir diese nur kurz berichten. Es zeigen sich insbesondere Einflüsse der elterlichen Bildung und des sozioökonomischen Status. Weitere signifikante Ergebnisse sind ein negativer Einfluss wirtschaftlicher Sorgen und ein positiver Einfluss des Alters der Mutter bei Geburt, wobei wir letzteres nur als Kontrollvariable berücksichtigen. Außerdem zeigen sich die Auswirkungen des vorherigen Bildungsverlaufs. Eine frühere Platzierung in gymnasialen Bildungsgängen steht erwartungsgemäß mit einer deutlich höheren $(\leftarrow$ p. 635) Studienwahrscheinlichkeit im Zusammenhang. Auf diese Ergebnisse werden wir im Folgenden nur noch indirekt eingehen. Da es sich um Faktoren handelt, die mit dem Arbeitslosigkeitsrisiko korrelieren oder über die der Einfluss der Arbeitslosigkeit auf den Bildungserfolg vermittelt wird, führt die Verwendung entsprechender Variablen in einer stufenweisen Modellierung zu einer Veränderung der Koeffizienten der Arbeitslosigkeitsvariablen, die entsprechend unserer $\mathrm{Hy}$ pothesen im zentralen Fokus der empirischen Analysen stehen.

\footnotetext{
${ }^{4}$ Analysen zur Validität der Skala deuten darauf hin, dass das zweite Item („Eltern sprechen Dinge an, die Befragte ärgern oder belasten") nicht in ausreichender Weise die gemessene Dimension abbildet, sodass wir es bei der Indexbildung nicht berücksichtigen (vgl. auch Weinhardt und Schupp 2011: 21).

${ }^{5}$ Wie in Abschnitt 3 erwähnt haben wir auch logistische Regressionsmodelle geschätzt, deren Ergebnisse sich kaum von den hier dargestellten unterscheiden. So gibt es bei 5-Prozent-Irrtumswahrscheinlichkeit bei den in Tabelle A2 aufgeführten signifikanten Koeffizienten nur zwei Abweichungen, wenn anstelle eines linearen Wahrscheinlichkeitsmodells ein logistisches Regressionsmodell geschätzt wird. Beide Abweichungen betreffen Modell 6 , sind aber für die Schlussfolgerungen aus den Analysen nicht zentral. Der positive Koeffizient für "Universität“ als höchster Bildungsabschluss der Eltern ist im Fall der logistischen Regression nicht signifikant. Der Koeffizient „große wirtschaftliche Sorgen (Ende der Sekundarschulzeit)“ ist im logistischen Modell signifikant, während er dies im linearen Wahrscheinlichkeitsmodell nicht ist.
} 
Wie erwartet ( $\mathrm{H} 1)$ zeigt sich in Modell 1 jeweils ein negativer Einfluss der Arbeitslosigkeit, wobei nur das mehrfache Auftreten von Arbeitslosigkeit während der Sekundarschulzeit signifikant die Wahrscheinlichkeit verringert, ein Studium zu beginnen. Gemessen an der Studienwahrscheinlichkeit in der Referenzpopulation (36,4 Prozent, siehe Modellkonstante) hat die mehrfache väterliche Arbeitslosigkeit einen sehr stark negativen Einfluss (minus 20,7 Prozentpunkte). Die Ergebnisse bestätigen auch die Erwartungen zur Häufigkeit des Auftretens bzw. der Dauer von Arbeitslosigkeit und der Zeitlichkeit (H4a/H4b). Länger zurückliegende Arbeitslosigkeit weist keinen signifikanten Einfluss auf. Gleiches gilt für einmalige Arbeitslosigkeitserfahrungen. ${ }^{6}(\leftarrow$ p. 636)

Die Berücksichtigung von Kontrollvariablen verändert die Ergebnisse nur geringfügig (Modell 2). Deutlichere Unterschiede zeigen sich in Modell 3, das die elterliche Bildung und den sozioökonomischen Status enthält. Der zuvor deutlich negative Einfluss der Arbeitslosigkeit ist zum Teil auf die soziale Selektivität des Arbeitslosigkeitsrisikos zurückzuführen. Allerdings ist auch nach Kontrolle der herkömmlichen Statusmerkmale ein signifikant negativer Zusammenhang zwischen Arbeitslosigkeit und Studienwahrscheinlichkeit zu beobachten ( $\mathrm{H} 2)$. Die zusätzliche Aufnahme des Einkommens (Modell 4) und der wirtschaftlichen Sorgen (Modell 5) verändert die Ergebnisse kaum. Während das Einkommen keinen über die Bildung und den sozio-ökonomischen Status hinausgehenden Einfluss aufweist $(\mathrm{H} 3 \mathrm{a})$, verringern wirtschaftliche Sorgen der Eltern signifikant die Wahrscheinlichkeit ein Studium zu beginnen (H3c). Wie der Vergleich der Ergebnisse der Modelle 4 und 5 in Abbildung 1 zeigt, wird der Einfluss der Arbeitslosigkeit über diese familialen Faktoren kaum vermittelt.

Die bisherigen Ergebnisse zeigen, dass insbesondere die soziale Selektivität der Arbeitslosigkeit eine entscheidende Rolle spielt. Gleichzeitig wird aber auch deutlich, dass auch bei Berücksichtigung einer Vielzahl von Kontroll- und Statusvariablen sowie vermittelnder Faktoren das mehrfache Auftreten von väterlicher Arbeitslosigkeit weiterhin signifikant negativ auf die Wahrscheinlichkeit wirkt, ein Studium zu beginnen. Dies ändert sich erst, wenn die Platzierung im Bildungssystem zu Beginn und zum Ende der Sekundarschulzeit ins Modell aufgenommen und somit Aspekte der institutionellen Bedingtheit des Bildungsverlaufs berücksichtigt werden (Modell 6). Somit weist die väterliche Arbeitslosigkeit keinen direkten Einfluss auf die Studienwahrscheinlichkeit auf. Es besteht aber möglicherweise ein Zusammenhang mit einer früheren Platzierung im Bildungssystem, die einen Übergang ins Studium unwahrscheinlicher macht.

\footnotetext{
${ }^{6}$ Wir vergleichen das Auftreten des Ereignisses Hochschulstudium gegen eine relativ heterogene Referenzkategorie. Wir haben zusätzlich Analysen mit einer homogeneren Referenzkategorie durchgeführt (Personen mit Abitur, die kein Studium begonnen haben). Die Ergebnisse sind mit den hier berichteten vergleichbar. Allein bei mehrfacher väterlicher Arbeitslosigkeit sind signifikante Einflüsse beobachtbar. Die Effektstärke ist etwas höher, die Koeffizienten sind aber bereits ab Modell 3 nicht mehr auf 5\%-Niveau signifikant, was auf die geringere Fallzahl zurückzuführen sein dürfte (Ergebnisse nicht dargestellt).
} 
Wir werden in Abschnitt 6 den Einfluss der Arbeitslosigkeit auf frühere Bildungsergebnisse untersuchen und dabei auch noch einmal auf die Ergebnisse von Modell 6 eingehen.

Abbildung 2 enthält die entsprechenden Ergebnisse zum Einfluss der mütterlichen Arbeitslosigkeit. Diese scheint nicht nur einen schwächeren Einfluss als die väterliche Arbeitslosigkeit zu haben $(\mathrm{H} 4 \mathrm{c})$, sondern es zeigen sich insgesamt keine signifikanten negativen Einflüsse, auch wenn die Koeffizienten für den Fall der mehrfach auftretenden Arbeitslosigkeit erwartungsgemäß negativ sind. Dies liegt nur in geringem Maße daran, dass in den Modellen die Arbeitslosigkeit des Vaters und der Mutter gleichzeitig betrachtet werden. Die Ergebnisse zusätzlicher Modelle (nicht dargestellt), in denen ausschließlich Variablen zur mütterlichen Arbeitslosigkeit enthalten sind, weichen nicht grundlegend von den in Abbildung 2 berichteten Ergebnissen ab. Für die mütterliche Arbeitslosigkeit zeigen sich dabei etwas stärker ausgeprägte Muster, signifikante Koeffizienten sind aber auch in diesen Modellen nicht zu beobachten. Hinsichtlich des grundlegenden Musters bekräftigen die Ergebnisse zur Arbeitslosigkeit der Mutter die zuvor diskutierten Ergebnisse zur Arbeitslosigkeit des Vaters. Wenn es einen negativen Einfluss der Arbeitslosigkeit gibt, tritt dieser auf, wenn die Arbeitslosigkeit $(\leftarrow$ p. 637) zeitlich näher an dem betrachteten Bildungsergebnis liegt und wenn Arbeitslosigkeit mehrfach bzw. dauerhaft auftritt. Dass die Ergebnisse der Modelle, in denen nur die mütterliche Arbeitslosigkeit enthalten ist, hinsichtlich der Effektstärke leicht von den hier präsentierten Ergebnissen abweichen, verweist auf Statushomogamie, die sich auch in einem erhöhten Arbeitslosigkeitsrisiko jeweils beider Elternteile zeigt. Da letztlich vor allem die Arbeitslosigkeit des Vaters in relevanter Weise auf den Bildungserfolg wirkt, werden wir im Folgenden ausschließlich diese betrachten.

\section{Ergebnisse weiterer Analysen}

Die in Abschnitt 5 dargestellten Ergebnisse bestätigen die Hypothese zum Zusammenhang zwischen Arbeitslosigkeit und Studienwahrscheinlichkeit, auch wenn nicht jede Form des Auftretens von Arbeitslosigkeit für den Bildungserfolg relevant ist. Sie zeigen aber auch, dass die Berücksichtigung sozialer Selektivität, familialer Faktoren und insbesondere des vorherige Bildungsverlaufes den Zusammenhang erklären oder vermitteln, sodass im abschließenden Modell kein direkter signifikanter Arbeitslosigkeitseinfluss mehr zu beobachten war. In diesem Abschnitt werden wir die bisherigen Analysen in drei Richtungen ergänzen bzw. vertiefen. Erstens haben wir als mögliche familiale Mediatoren bislang vor allem ökonomische Faktoren betrachtet. In zusätzlichen Analysen auf Basis eines anderen Samples werden wir auch $(\leftarrow \mathbf{p}$. 638) den Einfluss der familialen Beziehungen berücksichtigen $(\mathrm{H} 3 b)$. Zweitens hat sich in den 
bisherigen Analysen erwartungsgemäß die frühere Platzierung im Bildungssystem als besonders relevant erwiesen, was auf die institutionelle Abhängigkeit des Bildungsverlaufs verweist. Um zu klären, inwieweit Arbeitslosigkeitsepisoden frühere Bildungsergebnisse beeinflussen und diese dann auf spätere Bildungsübergänge nachwirken $(\mathrm{H} 3 \mathrm{~d})$, betrachten wir in zusätzlichen Analysen die Wahrscheinlichkeit des Gymnasialbesuches in der Sekundarschulzeit. Drittens können wir trotz der Berücksichtigung eines umfangreichen Sets an inhaltlichen Variablen und Kontrollvariablen nicht ausschließen, dass der mögliche Einfluss der väterlichen Arbeitslosigkeit auf andere, nicht im Modell beobachtete Variablen zurückzuführen und somit nicht kausal zu interpretieren ist. Ergänzend berichten wir daher die Ergebnisse von fixed effects Modellen, in denen wir die Variation zwischen Geschwistern nutzen, um den Einfluss konstanter unbeobachtbarer Faktoren ausschließen zu können (siehe Abschnitt 4).

Tabelle 2 enthält ausgewählte Ergebnisse aus Modellen auf Basis einer Stichprobe, für die auch Angaben zum elterlichen Unterstützungsverhalten und zu Schulleistungen (Mathematiknote, Klassenwiederholung) im letzten Schuljahr bzw. im Alter von 17 Jahren vorliegen. Modell $1 \mathrm{~b}$ in der Tabelle entspricht den in Abschnitt 5 dargestellten Modellen, die allein Arbeitslosigkeitsvariablen der Eltern enthalten. Auch hier zeigt sich der negative Zusammenhang zwischen mehrfacher bzw. längerer väterlicher Arbeitslosigkeit während der Sekundarschulzeit und der Studienwahrscheinlichkeit. Die in Modell $2 b$ hinzugefügten Variablen verändern das Ergebnis $(\leftarrow$ p. 639) nur geringfügig. Es zeigt sich aber in erwartbarer Weise, dass insbesondere die Schulleistungen auf die Wahrscheinlichkeit des Studienbeginns einwirken. Die leichte Verringerung der Stärke des Arbeitslosigkeitseffekts ist darauf zurückzuführen, dass die Schulleistungen von Kindern von Arbeitslosen schlechter ausfallen, was als primärer Herkunftseffekt zu interpretieren wäre. Es ist aber weniger ein direkter Einfluss der Arbeitslosigkeit, sondern eher Ausdruck der dahinterliegenden Merkmale wie elterliche Bildung und des sozio-ökonomischer Status. Es ist die Berücksichtigung dieser und weiterer Faktoren in den Modellen 3b und $4 \mathrm{~b}$ (die den vollständigen Modellen des vorherigen Abschnitts entsprechen), die auf die Stärke und Signifikanz des Arbeitslosigkeitseinflusses einwirken. Nach Kontrolle anderer Statusmerkmale ist der Zusammenhang zwischen Arbeitslosigkeit und Schulleistungen annährend zu vernachlässigen. Noch stärker gilt dies für das elterlichen Unterstützungsverhalten, das als ein Indikator der Qualität familialen Beziehungen berücksichtigt wurde. Auf Basis der vorliegenden Ergebnisse muss die entsprechende Hypothese $(\mathrm{H} 3 \mathrm{~b})$ abgelehnt werden.

Analog zu den in Abschnitt 5 dargestellten Analysen zur Wahrscheinlichkeit des Studienbeginns haben wir weitere Analysen durchgeführt, in denen wir die Wahrscheinlichkeit untersuchen, zu Beginn (erstes Jahr nach Grundschule) bzw. zum Ende der Sekundarschulzeit ein 
Gymnasium zu besuchen. Abbildung 3 fasst die Ergeb- ( $\leftarrow$ p. 640) nisse dieser Analysen zusammen. Im Gegensatz zum vorherigen Vorgehen schätzen wir nur fünf Modelle zur Bildungsbeteiligung zu Beginn der Sekundarschulzeit, da der frühere Bildungsverlauf hier nicht relevant ist. Modell 6 der Analysen zur Bildungsbeteiligung zum Ende der Sekundarschulzeit enthält wie bisher Angaben zur Platzierung zu Beginn der Sekundarschulzeit. Die Ergebnisse bestätigen allgemein die zuvor beobachteten Muster. Die Wahrscheinlichkeit, zu Beginn der Sekundarschulzeit ein Gymnasium zu besuchen, ist um 10,9 Prozentpunkte geringer, wenn der Vater zuvor in mehreren Jahren arbeitslos war (Modell 1). Am Ende der Sekundarschulzeit ist die Wahrscheinlichkeit sogar um 24,6 Prozentpunkte geringer, wenn der Vater zuvor in mehreren Jahren arbeitslos war. Im Unterschied zu den vorherigen Analysen wirkt auch die einmalige Arbeitslosigkeit während der Sekundarschulzeit signifikant negativ, allerdings erwartungsgemäß schwächer als die mehrfache Arbeitslosigkeit. Arbeitslosigkeitsepisoden während der Grundschulzeit spielen dagegen keine Rolle. Die Ergebnisse zeigen deutlich, dass Arbeitslosigkeitsepisoden während der Sekundarschulzeit für den weiteren Bildungsverlauf kritisch sind. Der im Vergleich zum Einfluss der Arbeitslosigkeit zu Beginn der Sekundarschulzeit deutlich negativere und vor allem über alle Modelle hinweg signifikante Einfluss bedeutet, dass Arbeitslosigkeit während der Sekundarschulzeit dazu führt, einen eingeschlagenen gymnasialen Bildungsweg zu verlassen oder vorzeitig zu beenden.

Zwar ist auch der Einfluss der Arbeitslosigkeit auf die Bildungsbeteiligung in der Sekundarschulzeit teilweise auf die soziale Selektivität des Arbeitslosigkeitsrisikos zurückzuführen. Die Berücksichtigung der elterlichen Bildung und des sozio-ökonomischen Status (Modell 3) erklärt wie in den vorherigen Analysen einen Teil des Arbeitslosigkeitseinflusses. Weiterhin besteht aber ein signifikant negativer Einfluss der Arbeitslosigkeit auf die Wahrscheinlichkeit, ein Gymnasium zum Ende der Schulzeit zu besuchen. Anders als in den Analysen zum Studienbeginn bleibt dieser Einfluss auch in weiteren Modellen bestehen, einschließlich von Modell (M6), das Angaben zur Bildungsbeteiligung zu Beginn der Sekundarschulzeit enthält. Dieses Ergebnis bestätigt die in Abschnitt 5 dargestellten Analysen, die deutlich gemacht haben, dass die Platzierung zum Ende der Sekundarschulzeit ein entscheidender Faktor ist, über den Arbeitslosigkeit auf die Studienwahrscheinlichkeit wirkt. Mit Blick auf nachschulische Bildungsereignisse wirkt die elterliche Arbeitslosigkeit über den vorherigen Bildungsverlauf nach.

Abschließend wollen wir auf das Problem eingehen, dass der Einfluss der Arbeitslosigkeit trotz der Berücksichtigung eines umfangreichen Sets weiterer Variablen auf andere, nicht im Modell beobachtete Faktoren zurückzuführen sein kann und somit nicht kausal zu interpretieren wäre. Abbildung 4 zeigt die Ergebnisse von Modellen, in denen wir das Vorhandensein von Geschwistern im Datensatz für fixed effects Schätzungen nutzen. Insbesondere bei mehrfacher 
Arbeitslosigkeit des Vaters in der Sekundarschulzeit war in den bisher durchgeführten Analysen ein negativer Einfluss auf die Wahrscheinlichkeit zum Ende der Schulzeit ein Gymnasium zu besuchen oder bis zum Alter von 23 Jahren ein Studium begonnen zu haben, zu beobachten. Die Abbildung enthält die Koeffizienten und Konfidenzintervalle für jeweils ein Modell, das nur die Arbeitslosigkeitsvariablen enthält (M1c) und eines, in dem zusätzlich zentrale Variablen enthalten sind, die zwischen Geschwistern Varianz aufweisen (M2c). In der oberen Hälfte der Abbildung sind Ergebnisse von $(\leftarrow$ p. 641) Analysen dargestellt, die auf derselben Stichprobe basieren wie die der in Abschnitt 5 durchgeführten Analysen. Die Schätzung beruht aber allein Personen, für die wir Geschwister beobachten $(n=470)$. Im unteren Teil der Abbildung sind Ergebnisse von Analysen dargestellt, in der wir die Stichprobe dadurch vergrößert haben, dass wir auf die Berücksichtigung von Variablen zu Beginn der Sekundarschulzeit oder nach Beendigung der Sekundarschulzeit verzichtet haben, wodurch sich die Fallzahlen vergrößern $(n=600$ bzw. $n=992)$.

Die Analysen zeigen einen deutlich negativen Einfluss der Arbeitslosigkeit auf die Wahrscheinlich, zum Ende der Sekundarschulzeit einen gymnasialen Bildungsgang zu besuchen und untermauern somit die in Abbildung 3 dargestellten Ergebnisse. Insbesondere in den Analysen auf Basis der kleineren Stichprobe ist der Koeffizient sehr groß, und dies obwohl durch die fixed effects Schätzung bereits sämtliche konstanten Familienmerkmale ,kontrolliert' wurden. Wie der Vergleich von Modell 1c und Modell 2c deutlich macht, verändert die zusätzliche Berücksichtigung von Merkmalen, in denen sich Geschwister unterscheiden können, die Ergebnisse nur geringfügig. Auch bei Kontrolle aller in den vorherigen Modellen verwendeten Variablen bleibt also ein stark negatives Ergebnis bestehen. Die im Vergleich zu den in Abschnitt 5 berichteten Ergebnissen enorme Effektstärke sollte aber nicht überinterpretiert werden, da aufgrund der geringen Effizienz der Schätzung die Kon- $(\leftarrow$ p. 642) fidenzintervalle sehr breit sind. So reicht dieses beispielsweise in Modell 2c zum Gymnasialbesuch bei einem Koeffizienten von $-0,228$ von $-0,444$ bis $-0,012$ (Irrtumswahrscheinlichkeit 5 Prozent). ${ }^{7}$

Es ist dagegen kein signifikanter Einfluss der Arbeitslosigkeit auf die Wahrscheinlichkeit des Studienbeginns festzustellen, und dies auch bereits in Modellen (M1c), die keine Angaben zum vorherigen Bildungsverlauf enthalten. Zwar verändert sich der relevante Arbeitslosigkeitskoeffizient, wenn familiale und bildungsverlaufsbezogene Faktoren berücksichtigt werden (M2c), was auf soziale Selektivität und mögliche Mediation verweist. Allerdings sind die jewei-

\footnotetext{
${ }^{7}$ Zum einen ist dies auf die geringe Fallzahl von 208 Familien mit Geschwistern, die über den Untersuchungszeitraum beobachtet wurden, zurückzuführen. Zum anderen unterscheidet sich in 71,2 Prozent dieser Familien das Bildungsergebnis der Geschwister nicht. Die Identifikation der Koeffizienten beruht also auf nur 60 Familien, in denen Varianz in der abhängigen Variablen vorliegt.
} 
ligen Koeffizienten in den Modellen zur Studienwahrscheinlichkeit sämtlich insignifikant, sodass wir diese Veränderungen nicht weiter interpretieren wollen. Bei ausschließlicher Betrachtung der Ergebnisse der fixed effects Modelle muss die Hypothese $(\mathrm{H} 2)$, dass Arbeitslosigkeit einen über familiale Statusmerkmale hinausgehenden Einfluss auf die Studienwahrscheinlichkeit aufweist, verworfen werden. Für die Wahrscheinlichkeit des Gymnasialbesuches wird dagegen ein solcher Einfluss identifiziert.

\section{Diskussion und Ausblick}

Im vorliegenden Papier haben wir die Frage untersucht, ob elterliche Arbeitslosigkeit einen Einfluss auf den Bildungserfolg von Kindern ausübt. Dass Arbeitslosigkeit und Bildungserfolg negativ korrelieren, hat sich in vielen früheren Studien gezeigt und bestätigt sich auch in unseren Ergebnissen. Darüber hinaus konnten wir zeigen, dass in Zwei-Elternfamilien allein die väterliche Arbeitslosigkeit einen einschneidenden Einfluss auf Bildungsergebnisse aufweist und dies auch nur dann, wenn sie mehrfach bzw. längerfristig auftritt. Auch der Zeitpunkt der Arbeitslosigkeit spielt eine Rolle. Insbesondere Arbeitslosigkeit während der Sekundarschulzeit hat negative Auswirkungen, und diese wirkt direkt auf den Schulbesuch und indirekt auf das von uns betrachtete spätere Bildungsergebnis eines ,Studienbeginns'. Somit konnten die allgemein formulierte Hypothese zum negativen Einfluss der Arbeitslosigkeit (H1) und die damit verbundenen Unterhypothesen $(\mathrm{H} 4 \mathrm{a}-\mathrm{c})$ bestätigt werden. Zudem konnten wir zeigen, dass der Arbeitslosigkeitseinfluss über familiale Faktoren wie insbesondere die wahrgenommene ökonomische Unsicherheit vermittelt ist $(\mathrm{H} 3 \mathrm{c})$. Eine Mediation über das Einkommen $(\mathrm{H} 3 \mathrm{a})$ konnten wir kaum beobachten, was teilweise auf die gleichzeitige Berücksichtigung von Bildung und sozio-ökonomischem Status zurückzuführen ist. Keine Unterstützung konnten wir für die Hypothese finden ( $\mathrm{H} 3 \mathrm{~b})$, dass ein problematischeres Familienklima als ursächliche Folge von Arbeitslosigkeit für den geringeren Bildungserfolg verantwortlich ist, wobei wir nur auf einmalige retrospektive Angaben zum Familienklima zurückgreifen konnten und nicht auf dynamische Informationen. $(\leftarrow$ p. 643)

Für die Interpretation der Ergebnisse ist jedoch die Frage wichtiger, ob Arbeitslosigkeit auch über stabile Merkmale der sozialen Herkunft hinaus, die sowohl auf das Arbeitslosigkeitsrisiko als auch auf den Bildungserfolg wirken, einen Einfluss aufweist. Die Hypothese, dass Arbeitslosigkeit einen über andere Herkunftsmerkmale hinausgehenden Einfluss auf den Bildungserfolg aufweist $(\mathrm{H} 2)$, kann auf Basis unserer Ergebnisse nicht vollends bestätigt, aber auch nicht ohne weiteres verworfen werden. Auch bei umfangreicher Kontrolle weiterer Variablen zeigt sich ein über den früheren Bildungsverlauf vermittelter Einfluss der mehrfach bzw. längerfristig 
auftretenden Arbeitslosigkeit des Vaters während der Sekundarschulzeit auf die Studienentscheidung. Ein weiteres Ergebnis ist, und dies auch bei Berücksichtigung konstanter unbeobachteter Heterogenität im Rahmen von fixed effects Modellen, dass Arbeitslosigkeit die Wahrscheinlichkeit erhöht, einen bereits eingeschlagenen gymnasialen Bildungsweg zu verlassen oder vorzeitig zu beenden. Zusammen mit dem Befund der Vermittlung über frühere Bildungsergebnisse spricht dies für einen über stabile Herkunftsmerkmale hinausgehenden Einfluss der Arbeitslosigkeit. Gleichzeitig sind die Ergebnisse der fixed effects Modelle zu den unterschiedlichen Bildungsergebnissen widersprüchlich, sodass die Ergebnisse diese Interpretation nicht vollends unterstützen: Zwar wirkt Arbeitslosigkeit negativ auf den Gymnasialbesuch, damit geht aber keine - eigentlich zu erwartende - Verringerung der Studienwahrscheinlichkeit einher. Wie bereits angesprochen, muss dieses Ergebnis vor dem Hintergrund der geringen Effizienz der fixed effects Schätzungen vorsichtig interpretiert werden. Eindeutig ist aber das Ergebnis, dass auch bei Kontrolle umfangreicher Merkmale bzw. im Rahmen von fixed effects Modellen ein Einfluss einer mehrfach auftretenden väterlichen Arbeitslosigkeit auf Bildungsergebnisse zu beobachten ist.

Was bedeutet dies für die Frage, ob soziale Herkunft als stabiles oder dynamisches Merkmal aufgefasst werden sollte? Und welche sozial-, arbeitsmarkt- und bildungspolitischen Schlussfolgerungen ergeben sich daraus? Zur ersten Frage belegen unsere Analysen, dass Bildungsverläufe von familialen Ereignissen beeinflusst werden können, die somit einen zusätzlichen, wenn auch im Vergleich zu stabilen Statusmerkmalen weniger bedeutsamen Erklärungsbeitrag bieten. Mit Blick auf die zweite Frage können wir feststellen, dass das Auftreten von mehrfacher Arbeitslosigkeit Kinder von bereits eingeschlagenen höheren Bildungswegen ablenken können. Die Analysen haben zudem gezeigt, dass es insbesondere die mit der Arbeitslosigkeit verbundene ökonomische Unsicherheit ist, die dies bewirkt. Entsprechend könnten sozial- und arbeitsmarktpolitische Maßnahmen, die auf eine Bekämpfung von Arbeitslosigkeit sowie eine Verringerung der mit ihr einhergehenden Unsicherheit zielen, den negativen Auswirkungen auf den Bildungserfolg der Kinder von Arbeitslosen begegnen. Bildungspolitisch wäre diesem negativen Einfluss durch eine von den Eltern unabhängige Finanzierung des Besuchs von höheren Bildungswegen zu begegnen. Darüber hinaus belegen unsere Analysen einmal mehr die auch indirekten negativen Konsequenzen mehrgliedriger Sekundarschulsysteme. Der „frühe“ negative Einfluss der väterlichen Arbeitslosigkeit während der Sekundarschulzeit wirkt noch viele Jahre später auf die Studienwahrscheinlichkeit nach.

Abschließend möchten wir noch auf einige Faktoren bzw. Unterschiede eingehen, die wir in unseren bereits umfangreichen Analysen nicht oder nicht näher $(\leftarrow$ p. 644) berücksichtigt haben, auf die aber die Literatur zur Wirkung von elterlicher Arbeitslosigkeit verweist. Wir haben 
zu manchen dieser Fragen vorläufige Analysen angestellt, die wir hier kurz berichten möchten. US-amerikanische Studien enthalten Hinweise auf unterschiedliche Auswirkungen elterlicher Arbeitslosigkeit je nach Einkommen oder sozialem Status (Brand et al. 2014; Kalil 2013; Stevens und Schaller 2011). Die Berücksichtigung von Interaktionen zwischen elterlicher Bildung und Arbeitslosigkeit in vorläufigen Analysen führte zu nicht signifikanten Ergebnissen. Andere Studien thematisieren Geschlechterunterschiede des Kindes, also ob Söhne oder Töchter unterschiedlich auf die Arbeitslosigkeit von Vater und Mutter reagieren (Andersen 2013; Müller et al. 2017). Für Deutschland finden Müller et al. (2017) für Töchter einen unerwarteten, positiven Einfluss väterlicher Arbeitslosigkeit auf die Bildungsbeteiligung. Sie diskutieren unterschiedliche Mechanismen, wie dieser Befund erklärt werden könne (risikovermeidendes Verhalten, Heiratsmarktüberlegungen und mütterliche Rollenmodelle). Keiner dieser Mechanismen lässt sich in ihrer Studie empirisch überzeugend nachweisen. In vorläufigen Analysen finden wir einen positiven Einfluss sowohl mütterlicher als auch väterlicher einmaliger Arbeitslosigkeit auf die Wahrscheinlichkeit eines Studienbeginns von Töchtern, während der Einfluss bei Söhnen negativ ist. Für den Fall mehrfacher Arbeitslosigkeit finden wir dagegen keine unterschiedlichen geschlechtsspezifischer Muster. Um ausschließen zu können, dass es sich bei dem Ergebnis in der Studie von Müller et al. (2017) um ein Methodenartefakt handelt, wäre eine umfassendere Replikation aus unserer Sicht sehr hilfreich. Als Beschränkungen unserer Analysen aufgrund von Fallzahlproblemen haben wir bereits den Ausschluss von Ein-ElternFamilien und Ostdeutschland angesprochen. Gerade die Frage, ob Arbeitslosigkeitswirkungen sich in Ost- und Westdeutschland unterscheiden, erscheint vor dem Hintergrund der weiterhin bestehenden Arbeitsmarktunterschiede relevant. Hinweise auf die Wirkung von wendebedingten Arbeitslosigkeitserfahrungen in Ostdeutschland finden sich bei Becker und Nietfeld (1999). $(\leftarrow$ p. 645) 


\section{Literatur}

Andersen, Signe Hald. 2013. Common genes or exogenous shock? Disentangling the causal effect of paternal unemployment on children's schooling efforts. In European Sociological Review, 29(3):477-488.

Becker, Rolf. 2011. Entstehung und Reproduktion dauerhafter Bildungsungleichheiten. In Lehrbuch der Bildungssoziologie. Hrsg. Rolf Becker, 87-138. Wiesbaden: VS Verlag.

Becker, Rolf und Nietfeld, Markus. 1999. Arbeitslosigkeit und Bildungschancen von Kindern im Transformationsprozeß. Eine empirische Studie über die Auswirkungen sozioökonomischer Deprivation auf intergenerationale Bildungsvererbung. Kölner Zeitschrift für Soziologie und Sozialpsychologie, 51(1):55-79.

Boudon, Raymond. 1974. Education, opportunity, and social inequality. Changing prospects in Western society. New York: Wiley-Interscience.

Bourdieu, Pierre. 1983. Ökonomisches Kapital, kulturelles Kapital, soziales Kapital. In Soziale Ungleichheiten. Hrsg: Reinhard Kreckel, 183-198. Göttingen. Soziale Welt Sonderband 2: Schwartz.

Brand, Jennie E. und Simon Thomas. Juli 2014. Job displacement among single mothers: Effects on children's outcomes in young adulthood. In American Journal of Sociology, 119(4):955-1001.

Coelli, Michael B. 2011. Parental job loss and the education enrollment of youth. In Labour Economics, 18(1):25-35.

Dronkers, Jaap. 1994. Father's unemployment and childrens academic achievement. In Education and Society, 12(1):3-21.

Ermisch, John, Marco Francesconi und David J. Pevalin. 2004. Parental partnership and joblessness in childhood and their influence on young people's outcomes. In Journal of the Royal Statistical Society, Series A (General), 167(1):69-101.

Grabka, Markus M. 2016. SOEP 2014 - Codebook for the \$PEQUIV File 1984-2014: CNEF Variables with Extended Income Information for the SOEP. SOEP Survey Papers 300: Series D. Berlin: DIW/SOEP.

Jonsson, Jan O. und Michael Gähler. 1997. Family dissolution, family reconstitution, and childern's educational careers: Recent evidence for Sweden. In Demography, 34(2):277-293.

Kalil, Ariel. 2013. Effects of the great recession on child development. In The ANNALS of the American Academy of Political and Social Science, 630(1):232-250.

Kalil, Ariel und Patrick Wightman. 2011. Parental job loss and children's educational attainment in black and white middle-class families. In Social Science Quarterly, 92(1):57-78.

Kalil, Ariel und Kathleen M. Ziol-Guest. 2008. Parental employment circumstances and children's academic progress. In Social Science Research, 37(2):500-515.

Lauterbach, Wolfgang und Andreas Lange. 1998. Aufwachsen in materieller Armut und sorgenbealstetem Familienklima. Konsequenzen für den Schulerfolg von Kindern am Beispiel des Übergangs in die Sekundarstufe I. In Armut und soziale Ungleichheit bei Kindern, Hrsg. Mansel, Jürgen und Georg Neubauer. 106-128. Opladen: Leske+Budrich.

Levine, Philip B. 2011. How does parental unemployment affect children's educational performance? In Whither opportunity? Rising inequality, schools, and childrens life chances, Hrsg. Duncan, Greg J. und Fichard J. Murnane. 315-335. New York: Russell Sage Foundation

Lohmann, Henning, C. Katharina Spieß, Olaf Groh-Samberg und Jürgen Schupp. 2009. Analysepotenziale des Sozio-oekonomischen Panels (SOEP) für die empirische Bildungsforschung. In Zeitschrift für Erziehungswissenschaft, 12(2):252-280.

Lohmann, Henning und Sven Witzke. 2011. BIOEDU (Beta Version): Biographical Data on Educational Participation and Transitions in the German Socio-Economic Panel Study (SOEP). DIW Data Documentation, Berlin: DIW.

Maaz, Kai, Jürgen Baumert und Ulrich Trautwein. 2010. Genese sozialer Ungleichheit im institutionellen Kontext der Schule: Wo entsteht und vergrößert sich soziale Ungleichheit? In Bildungsentscheidungen, Zeitschrift für Erziehungswissenschaft, 
Sonderheft 12, Hrsg. Baumert, Jürgen, Kai Maaz und Ulrich Trautwein. Wiesbaden: VS Verlag.

Manski, Charles F., Gary D. Sandefur, Sara McLanahan und Daniel Powers. 1992. Alternative estimates of the effect of family structure during adolescens on high school graduation. In Journal of the American Statistical Association, 87(417):25-37.

McLanahan, Sara, Laura Tach und Daniel Schneider. 2013. The causal effects of father absence. In Annual Review of Sociology, 39:399-427.

Müller, Walter uns Reinhard Pollak. 2004. Weshalb gibt es so wenige Arbeiterkinder in Deutschlands Universitäten? In Bildung als Privileg? Erklärungen und Befunde zu den Ursachen der Bildungsungleichheit, Hrsg. Becker, Rolf und Wolfgang Lauterbach, 311352. Wiesbaden: VS Verlag.

Müller, Steffen, Regina T. Riphahn und Caroline Schwientek. 2017. Paternal unemployment during childhood: causal effects on youth worklessness and educational attainment. In Oxford Economic Papers 69(1):213-238.

Peter, Frauke H. 2013. Trick or treat? Maternal involuntary job loss and children's noncognitive skills. DIW Discussion Papers 1297.

Rogge, Benedikt (2013): Wie uns Arbeitslosigkeit unter die Haut geht. Identitätsprozess und psychische Gesundheit bei Statuswechseln. Konstanz: UVK.

Schwarz, Beate und Sabine Walper. 1997. Erziehung aus Sicht von Eltern und Kindern. Erste Erfahrungen mit den Instrumenten der 1. Erhebung. Berichte aus der Arbeitsgruppe „Familienentwicklung nach der Trennung“ \#19/97, München: Ludwig-MaximiliansUniversität.

Stevens, Ann H. und Jessamyn Schaller. 2011. Short-run effects of parental job loss on children's academic achievement. In Economics of Education Review 30(2):289-299.

Ström, Sara. 2003. Unemployment and families: a review of research. In Social Service Review 77(3):399-430.

Wagner, Gert G., Joachim R. Frick und Jürgen Schupp. 2007. The German Socio-Economic Panel Study (SOEP) - Scope, Evolution and Enhancements. In Schmollers Jahrbuch. Zeitschrift für Wirtschafts- und Sozialwissenschaften, 127(1):139-169.

Weinhardt, Michael und Jürgen Schupp 2011. Multi-Itemskalen im SOEP Jugendfragebogen. DIW Data Documentation 60, Berlin: DIW. 


\section{Abbildung 1: Arbeitslosigkeit des Vaters und Wahrscheinlichkeit eines Studienbe- ginns (lineare Wahrscheinlichkeitsmodelle, random-effects)}

1 mal arbeitslos, Alter 5-10 J.

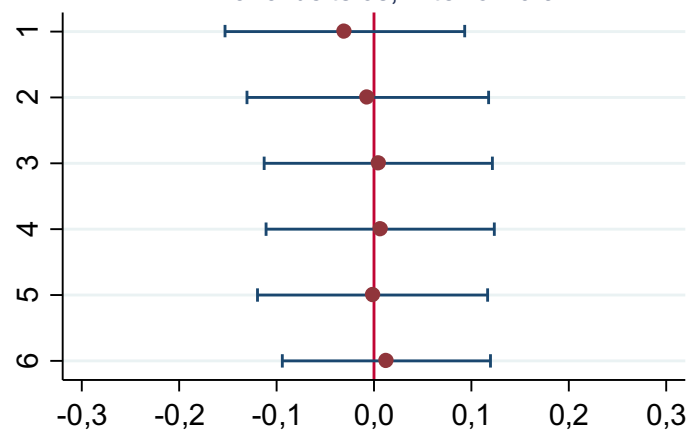

1 mal arbeitslos, Alter 11-17 J.

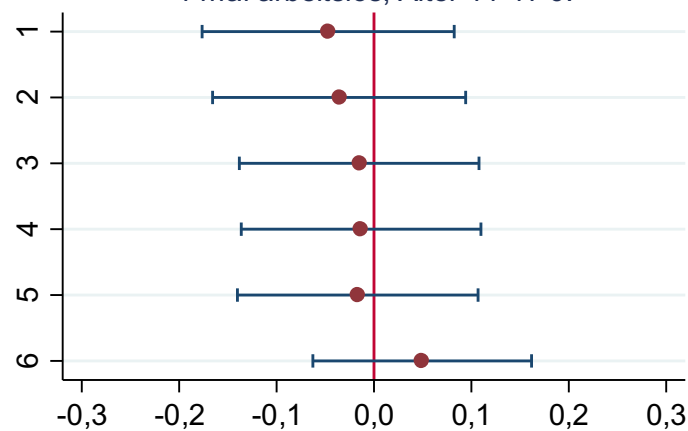

2+ mal arbeitslos, Alter 5-10 J.

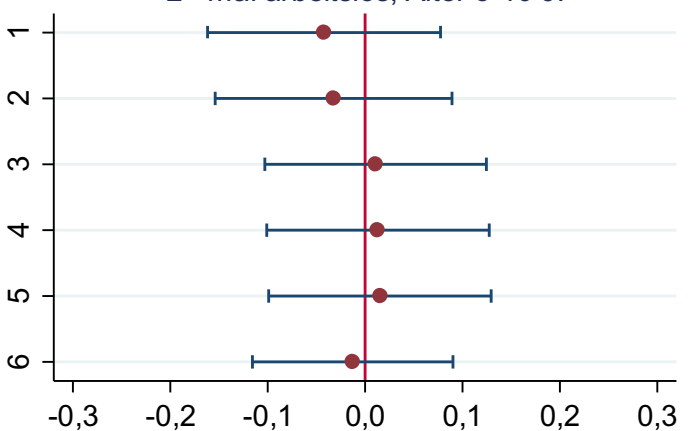

2+ mal arbeitslos, Alter 11-17 J.

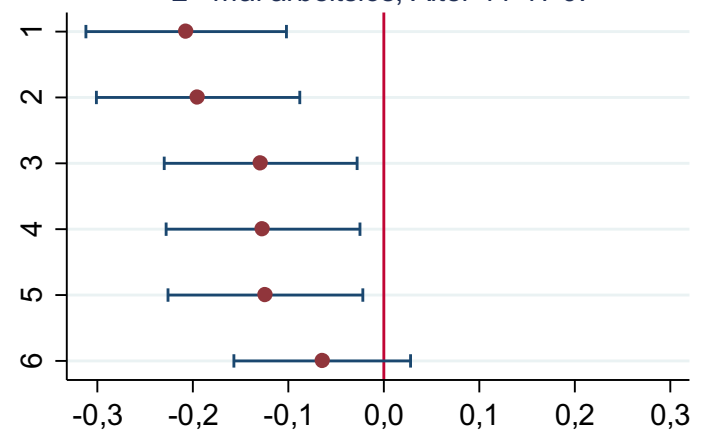

Koeffizient mit 95\%-Konfidenzintervall

Anmerkungen: SOEP v31.1 (ungewichtet, n=963), random effects Modelle berücksichtigen die Abhängigkeit von Kindern mit derselben Mutter, Erläuterung der Modelle in Tabelle 1, weitere Ergebnisse in Tabelle A2. 


\section{Abbildung 2: Arbeitslosigkeit der Mutter und Wahrscheinlichkeit eines Studienbeginns (lineare Wahrscheinlichkeitsmodelle, random effects)}
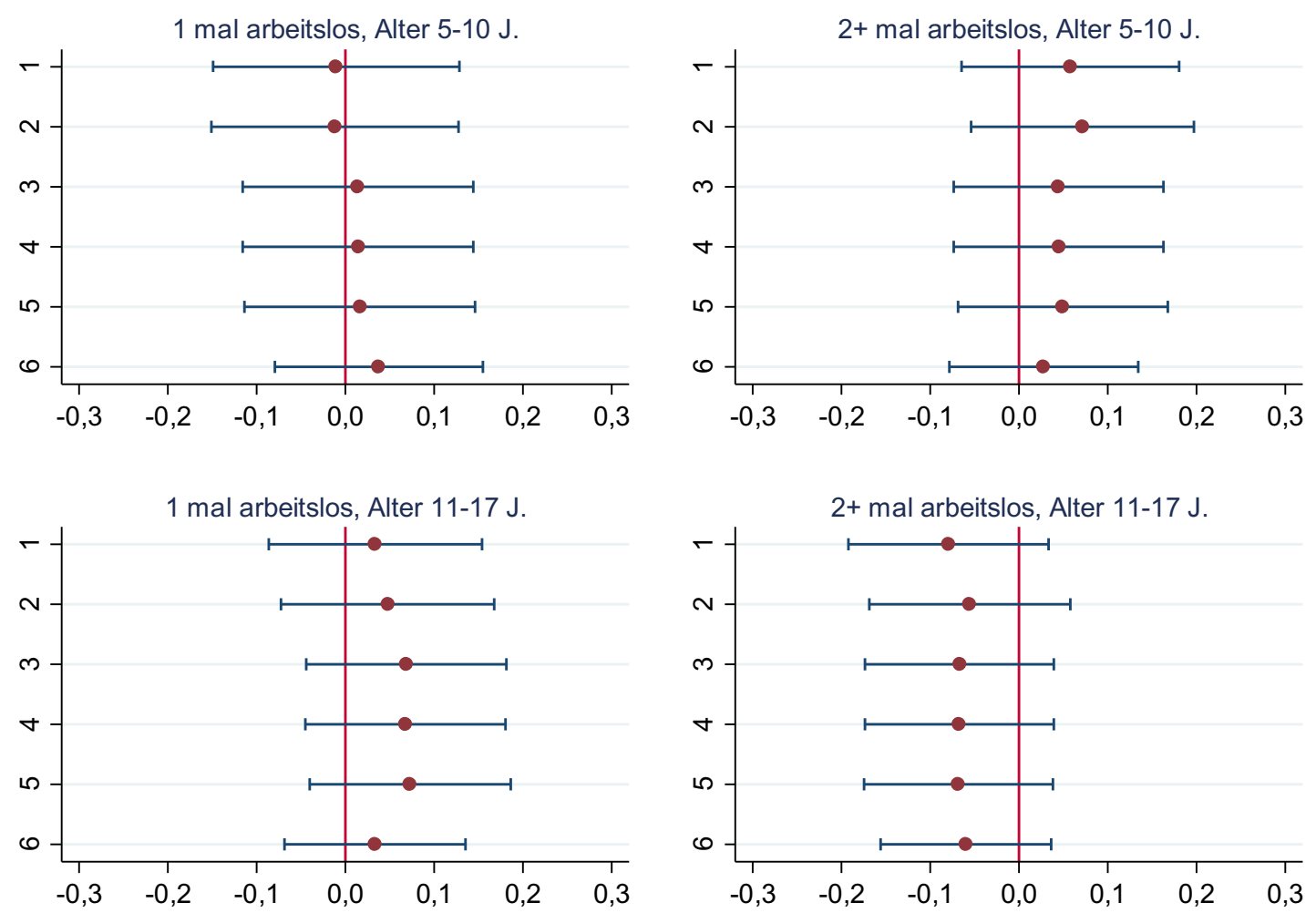

Koeffizient mit 95\%-Konfidenzintervall

Anmerkungen: SOEP v31.1 (ungewichtet, $n=963$ ), random effects Modelle berücksichtigen die Abhängigkeit von Kindern mit derselben Mutter, Erläuterung der Modelle in Tabelle 1, weitere Ergebnisse in Tabelle A2. 


\section{Abbildung 3: Arbeitslosigkeit des Vaters und Wahrscheinlichkeit des Gymnasialbe- suchs (lineare Wahrscheinlichkeitsmodelle, random effects)}

Beginn Sekundarstufe
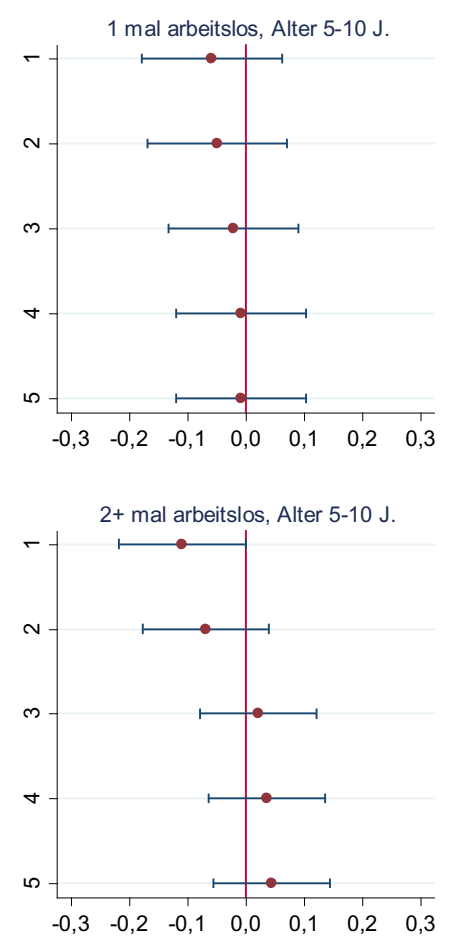

Ende Sekundarstufe
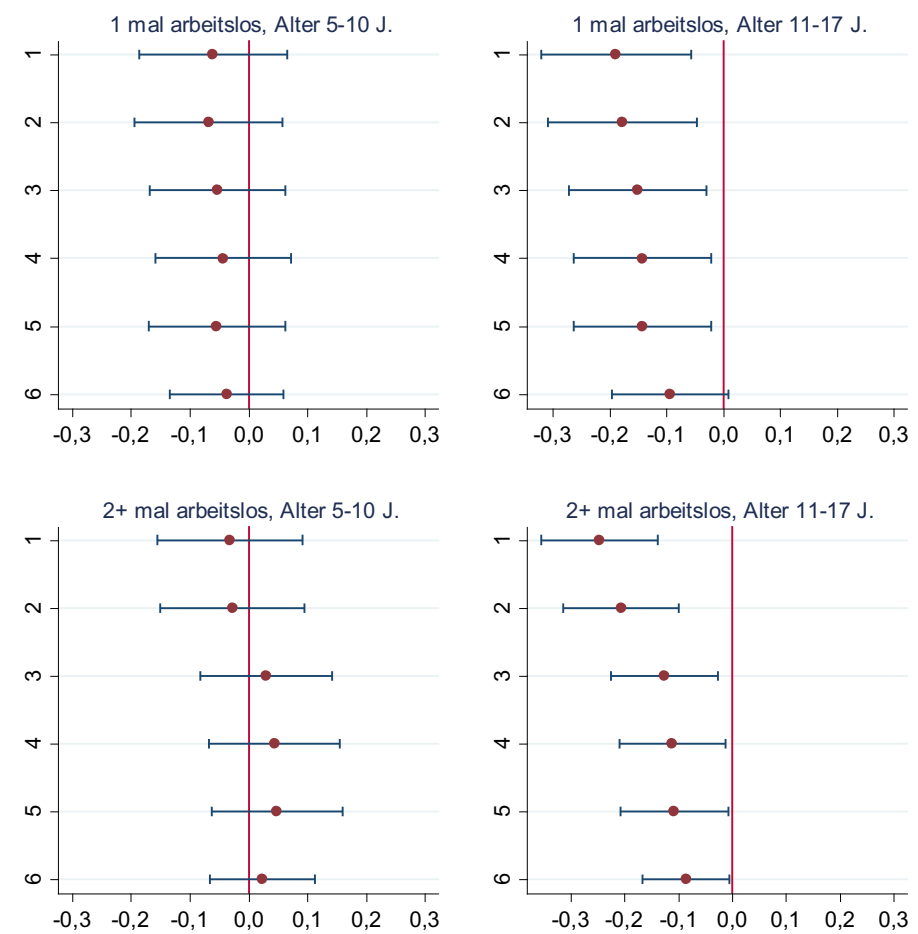

Koeffizient mit 95\%-Konfidenzintervall

Anmerkungen: SOEP v31.1 (ungewichtet, $n=963$ ), random effects Modelle berücksichtigen die Abhängigkeit von Kindern mit derselben Mutter, Erläuterung der Modelle in Tabelle 1. 


\section{Abbildung 4: Arbeitslosigkeit des Vaters und Wahrscheinlichkeit eines Studienbe- ginns bzw. Gymnasialbesuchs zum Ende der Schulzeit (lineare Wahrscheinlichkeits- modelle, fixed effects)}

Gymnasium (Ende Sek.) 1 mal arbeitslos, Alter 11-17 J.

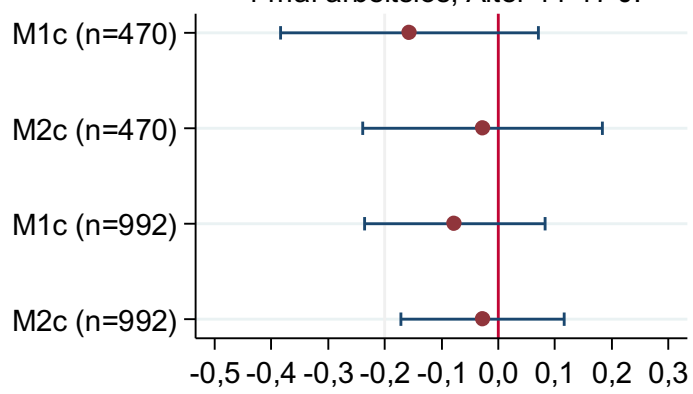

2+ mal arbeitslos, Alter 11-17 J.

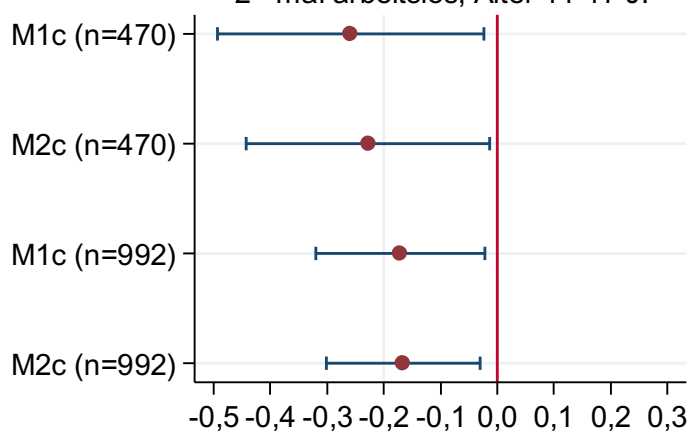

Beginn Studium

1 mal arbeitslos, Alter $11-17 \mathrm{~J}$
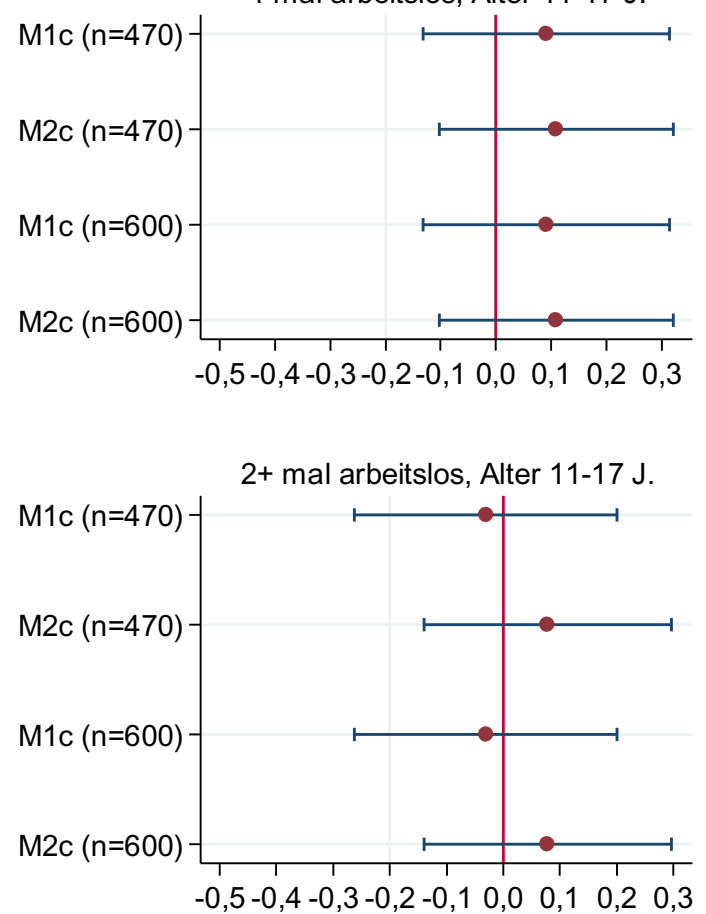

Koeffizient mit 95\%-Konfidenzintervall

Anmerkungen: SOEP v31.1 (ungewichtet), fixed effects Modelle für Kindern mit derselben Mutter. M1c enthält wie Modell 1 in Tabelle 1 Arbeitslosigkeitsvariablen des Vaters und der Mutter, M2c enthält alle Variablen aus Modell 6 außer höchster Bildungsabschluss der Eltern, ISEI und Bundesland (andere Variablen teilweise nur zu einem Zeitpunkt gemessen). 
Tabelle 1: Variablen und Modelle

\begin{tabular}{|c|c|c|}
\hline Modell & Variable & Operationalisierung \\
\hline \multirow[t]{2}{*}{ M1 } & $\begin{array}{l}\text { Arbeitslosigkeitsphasen des } \\
\text { Vaters und der Mutter }\end{array}$ & $\begin{array}{l}\text { in einem Jahr, in zwei oder mehr Jahren (Ref.: } \\
\text { nicht arbeitslos), jeweils für den Zeitraum von } 5 \\
\text { bis } 10 \text { Jahren und } 11 \text { bis } 17 \text { Jahre des Alters des }\end{array}$ \\
\hline & \multicolumn{2}{|c|}{ Variablen zusätzlich zum jeweils vorherigen Modell: } \\
\hline \multirow[t]{7}{*}{ M2 } & Geschlecht des Kindes & weiblich (Ref.: männlich) \\
\hline & Migrationshintergrund des & ja (Ref.: nein) \\
\hline & Alter der Mutter bei Geburt & in Jahren \\
\hline & $\begin{array}{l}\text { Größe des elterlichen } \\
\text { Haushalts }\end{array}$ & $\begin{array}{l}\text { Anzahl Haushaltsmitglieder zu Beginn und Ende } \\
\text { der Sekundarschulzeit }\end{array}$ \\
\hline & $\begin{array}{l}\text { Umzug mit dem elterlichen } \\
\text { Haushalt }\end{array}$ & $\begin{array}{l}\text { bis zum Beginn und während der } \\
\text { Sekundarschulzeit: ja (Ref.: nein) }\end{array}$ \\
\hline & Bundesland & $\begin{array}{c}\text { zum Ende der Sekundarschulzeit (Ref.: } \\
\text { Schleswig-Holstein) }\end{array}$ \\
\hline & $\begin{array}{l}\text { Zeitpunkt des Übergangs in die } \\
\text { Sekundarstufe }\end{array}$ & $\begin{array}{c}\text { 1988-1991, 1922-1995, 1996-1999, 2000-2004 } \\
\text { (Ref.: 1984-1987) }\end{array}$ \\
\hline \multirow[t]{2}{*}{ M3 } & $\begin{array}{l}\text { höchster Bildungsabschluss } \\
\text { der Eltern }\end{array}$ & $\begin{array}{l}\text { Hauptschulabschl.+Ausb., mittl. Abschl.+Ausb., } \\
\text { (Fach)-Hochschulreife+Ausb., Fachhochschule, } \\
\text { Universität, Sonstige (Ref.: kein/ Hauptschul- } \\
\text { abschl.), höherer Wert des Vaters oder der }\end{array}$ \\
\hline & $\begin{array}{c}\text { sozio-ökonomischer Status der } \\
\text { Eltern }\end{array}$ & $\begin{array}{l}\text { durchschnittl. ISEI während Sekundarschulzeit, } \\
\text { jeweils höherer Wert des Vaters oder der Mutter }\end{array}$ \\
\hline M4 & $\begin{array}{l}\text { relative Einkommensposition } \\
\text { des elterlichen Haushalts }\end{array}$ & $\begin{array}{c}\text { durchschnittl. bedarfsgewichtetes } \\
\text { Haushaltsnettoeinkommen geteilt durch den } \\
\text { Median der Gesamtbevölkerung }\end{array}$ \\
\hline M5 & $\begin{array}{l}\text { wirtschaftliche Sorgen der } \\
\text { Eltern }\end{array}$ & $\begin{array}{l}\text { grosse Sorgen, einige Sorgen (Ref.: keine } \\
\text { Sorgen), jeweils höherer Wert des Vaters oder } \\
\text { der Mutter zu Beginn und Ende der }\end{array}$ \\
\hline \multirow[t]{2}{*}{ M6 } & $\begin{array}{l}\text { Schultyp zu Beginn und zum } \\
\text { Ende der Sekundarschulzeit }\end{array}$ & $\begin{array}{c}\text { Hauptschule/Hauptschulzweig, } \\
\text { Gymnasium/gymnasialer Zweig (Ref.: Andere) }\end{array}$ \\
\hline & \multicolumn{2}{|c|}{ Variablen in zusätzlichen Modellen auf Basis einer anderen Stichprobe: } \\
\hline $\mathrm{M} 3 / 4 \mathrm{~b}$ & Schulleistungen & $\begin{array}{c}\text { Mathematiknote mit } 17 \text { Jahren bzw. bei } \\
\text { Schulabschluss, Klassenwiederholung: ja (Ref. } \\
\text { nein) }\end{array}$ \\
\hline $\mathrm{M} 3 / 4 \mathrm{~b}$ & unterstützender Erziehungsstil & Indexwerte für Mutter und Vater \\
\hline
\end{tabular}


Tabelle 2: Wahrscheinlichkeit eines Studienbeginns (lineare Wahrscheinlichkeitsmodelle, random effects, Stichprobe mit Angaben aus Jugendfragebogen)

\begin{tabular}{|c|c|c|c|c|}
\hline & M1b & M2b & M3b & M4b \\
\hline \multicolumn{5}{|c|}{ Arbeitslosigkeit des Vaters (Alter 5-10 Jahre, Ref: nicht arbeitslos) } \\
\hline in einem Jahr & $-0,084$ & $-0,098$ & $-0,090$ & $-0,032$ \\
\hline in zwei oder mehr Jahren & $-0,020$ & 0,004 & 0,023 & 0,010 \\
\hline \multicolumn{5}{|c|}{ Arbeitslosigkeit des Vaters (Alter 11-17 Jahre, Ref: nicht arbeitslos) } \\
\hline in einem Jahr & $-0,091$ & $-0,037$ & $-0,041$ & 0,013 \\
\hline in zwei oder mehr Jahren & $-0,189 * * *$ & $-0,099+$ & $-0,092+$ & $-0,071$ \\
\hline \multicolumn{5}{|c|}{ unterstützender Erziehungsstil (Index): } \\
\hline Vater & & & $-0,004$ & $-0,012$ \\
\hline Mutter & & & 0,011 & $-0,013$ \\
\hline Schulleistungen: & & & 0,038 & 0,033 \\
\hline Note Mathematik & & & $-0,045 * * *$ & $-0,031^{* *}$ \\
\hline Klassenwiederholung & & & $-0,082 *$ & $-0,010$ \\
\hline \multicolumn{5}{|l|}{ Weitere Variablen: } \\
\hline Arbeitslosigkeit Mutter & ja & ja & ja & ja \\
\hline Bildung Eltern, ISEI & nein & ja & ja & ja \\
\hline Bildungsverlauf & nein & nein & nein & ja \\
\hline $\mathrm{s}_{\mathrm{e}}^{2}$ & 0,361 & 0,361 & 0,362 & 0,339 \\
\hline $\mathrm{s}_{\mathrm{u}}{ }^{2}$ & 0,250 & 0,196 & 0,187 & 0,104 \\
\hline $\mathrm{n}$ & 900 & 900 & 900 & 900 \\
\hline
\end{tabular}

Anmerkungen: SOEP v31.1 (ungewichtet), random effects Modelle berücksichtigen die Abhängigkeit von Kindern mit derselben Mutter, Signifikanzniveaus: $\left.\left.\left.{ }^{* * *}\right) 0,001,{ }^{* *}\right) 0,01,{ }^{*}\right) 0,05,+$ ) 0,1 . 
Tabelle A1: Variablenübersicht - univariate Statistiken

$\begin{array}{lllll}\text { Variable } & \text { arithm. Mittel Std.abw. } & \text { Min } & \text { Max }\end{array}$

Bildungsbeteiligung (Ref: kein Hochschulbesuch bzw. andere Schulform)

$\begin{array}{llll}\text { Hochschulbesuch } & 0,330 & 0,471 & 0\end{array}$

Hauptschule (Beginn Sek.)

$0,358 \quad 0,480$

Gymnasium (Beginn Sek.)

0,312

0,463

Hauptschule (Ende Sek.)

0,229

0,421

0,386

0,487

$\begin{array}{ll}0 & 1 \\ 0 & 1 \\ 0 & 1 \\ 0 & 1\end{array}$

Arbeitslosigkeit des Vaters (Alter 5-10 Jahre, Ref: nicht arbeitslos)

$\begin{array}{lllll}\text { ein Jahr } & 0,060 & 0,238 & 0 & 1 \\ \text { zwei oder mehr Jahre } & 0,090 & 0,287 & 0 & 1\end{array}$

Arbeitslosigkeit des Vaters (Alter 11-17 Jahre, Ref: nicht arbeitslos)

$\begin{array}{lllll}\text { in einem Jahr } & 0,052 & 0,222 & 0 & 1 \\ \text { in zwei oder mehr Jahren } & 0,116 & 0,321 & 0 & 1\end{array}$

Arbeitslosigkeit der Mutter (Alter 5-10 Jahre, Ref: nicht arbeitslos)

$\begin{array}{lllll}\text { in einem Jahr } & 0,051 & 0,220 & 0 & 1 \\ \text { in zwei oder mehr Jahren } & 0,067 & 0,251 & 0 & 1\end{array}$

Arbeitslosigkeit der Mutter (Alter 11-17 Jahre, Ref: nicht arbeitslos)

$\begin{array}{lllll}\text { in einem Jahr } & 0,065 & 0,247 & 0 & 1 \\ \text { in zwei oder mehr Jahren } & 0,080 & 0,271 & 0 & 1\end{array}$

Wirtschaftliche Sorgen der Eltern (Ref.: keine Sorgen)

$\begin{array}{lrrrr}\text { einige (Beginn Sek.) } & 0,272 & 0,445 & 0 & 1 \\ \text { grosse (Beginn Sek.) } & 0,531 & 0,499 & 0 & 1 \\ \text { einige (Ende Sek.) } & 0,321 & 0,467 & 0 & 1 \\ \text { grosse (Ende Sek.) } & 0,538 & 0,499 & 0 & 1 \\ \text { Ozio-ökonomischer Status } & & & & \\ \text { relative Einkommensposition } & 1,051 & 0,440 & 0,333 & 4,526 \\ \text { ISEl } & 45,298 & 15,526 & 16 & 88\end{array}$

höchster Bidungsabschluss der Eltern (Ref.: kein/Hauptschulabschluss)

\begin{tabular}{lllll} 
Hauptschulabschl.+Ausb. & 0,266 & 0,442 & 0 & 1 \\
mittl. Abschl.+Ausb. & 0,225 & 0,418 & 0 & 1 \\
(Fach)-Hochschulreife+Ausb. & 0,052 & 0,222 & 0 & 1 \\
Fachhochschule & 0,055 & 0,228 & 0 & 1 \\
Universität & 0,123 & 0,328 & 0 & 1 \\
Sonstige & 0,208 & 0,406 & 0 & 1 \\
mzüge (Ref.: nein) & & & & \\
während Grundschulzeit & 0,304 & 0,460 & 0 & 1 \\
während Sekundarschulzeit & 0,184 & 0,388 & 0 & 1 \\
\hline
\end{tabular}

Fortsetzung nächste Seite 
Fortsetzung von Tabelle A1

\begin{tabular}{lcccr}
\hline Bundesland (Ref.: Schleswig-Holstein) & & & & \\
Hamburg & 0,008 & 0,091 & 0 & 1 \\
Niedersachsen & 0,110 & 0,313 & 0 & 1 \\
Bremen & 0,006 & 0,079 & 0 & 1 \\
Nordrhein-Westfalen & 0,305 & 0,461 & 0 & 1 \\
Hessen & 0,070 & 0,255 & 0 & 1 \\
Rheinland-Pfalz/Saarland & 0,058 & 0,234 & 0 & 1 \\
Baden-Württemberg & 0,201 & 0,401 & 0 & 1 \\
Bayern & 0,183 & 0,387 & 0 & 1 \\
Berlin & 0,023 & 0,149 & 0 & 1 \\
Zeitpunkt des Übergangs in Sek.stufe (Ref.: $1984-1987)$ & & \\
1988-1991 & 0,214 & 0,410 & 0 & 1 \\
1922-1995 & 0,215 & 0,411 & 0 & 1 \\
1996-1999 & 0,177 & 0,381 & 0 & 1 \\
2000-2004 & 0,148 & 0,356 & 0 & 1 \\
Weitere Variablen & & & & \\
weiblich & 0,480 & 0,500 & 0 & 1 \\
Migrationshintergrund & 0,393 & 0,489 & 0 & 1 \\
$\quad$ Alter der Mutter bei Geburt & 27,163 & 5,247 & 16 & 49 \\
$\quad$ Haushaltsgröße (Beginn Sek.) & 4,575 & 1,249 & 2 & 12 \\
Haushaltsgröße (Ende Sek.) & 4,385 & 1,287 & 2 & 13 \\
\hline
\end{tabular}

Anmerkungen: SOEP v31.1 (ungewichtet) 
Tabelle A2: Wahrscheinlichkeit eines Studienbeginns (lineare Wahrscheinlichkeitsmodelle, random effects)

\begin{tabular}{|c|c|c|c|c|c|c|}
\hline & M1 & M2 & M3 & M4 & M5 & M6 \\
\hline \multicolumn{7}{|c|}{ Arbeitslosigkeit des Vaters (Alter 5-10 Jahre, Ref: nicht arbeitslos) } \\
\hline \multirow[t]{2}{*}{ ein Jahr } & $-0,030$ & $-0,006$ & 0,005 & 0,007 & $-0,001$ & 0,013 \\
\hline & 0,063 & 0,063 & 0,060 & 0,060 & 0,060 & 0,054 \\
\hline \multirow[t]{2}{*}{ zwei oder mehr Jahre } & $-0,042$ & $-0,032$ & 0,011 & 0,013 & 0,015 & $-0,013$ \\
\hline & 0,061 & 0,062 & 0,058 & 0,058 & 0,058 & 0,052 \\
\hline \multicolumn{7}{|c|}{ Arbeitslosigkeit des Vaters (Alter 11-17 Jahre, Ref: nicht arbeitslos) } \\
\hline \multirow[t]{2}{*}{ in einem Jahr } & $-0,047$ & $-0,035$ & $-0,015$ & $-0,013$ & $-0,016$ & 0,049 \\
\hline & 0,066 & 0,066 & 0,063 & 0,063 & 0,063 & 0,057 \\
\hline \multirow[t]{2}{*}{ in zwei oder mehr Jahren } & $-0,207 * * *$ & $-0,195 * * *$ & $-0,129$ * & $-0,127$ * & $-0,124$ * & $-0,065$ \\
\hline & 0,054 & 0,054 & 0,051 & 0,052 & 0,052 & 0,047 \\
\hline \multicolumn{7}{|c|}{ Arbeitslosigkeit der Mutter (Alter 5-10 Jahre, Ref: nicht arbeitslos) } \\
\hline \multirow[t]{2}{*}{ in einem Jahr } & $-0,010$ & $-0,012$ & 0,014 & 0,015 & 0,016 & 0,038 \\
\hline & 0,071 & 0,071 & 0,066 & 0,066 & 0,066 & 0,060 \\
\hline \multirow[t]{2}{*}{ in zwei oder mehr Jahren } & 0,058 & 0,071 & 0,044 & 0,045 & 0,049 & 0,028 \\
\hline & 0,063 & 0,064 & 0,060 & 0,060 & 0,060 & 0,054 \\
\hline \multicolumn{7}{|c|}{ Arbeitslosigkeit der Mutter (Alter 11-17 Jahre, Ref: nicht arbeitslos) } \\
\hline \multirow[t]{2}{*}{ in einem Jahr } & 0,034 & 0,048 & 0,069 & 0,068 & 0,073 & 0,033 \\
\hline & 0,061 & 0,061 & 0,058 & 0,058 & 0,058 & 0,052 \\
\hline \multirow[t]{2}{*}{ in zwei oder mehr Jahren } & $-0,079$ & $-0,056$ & $-0,067$ & $-0,067$ & $-0,069$ & $-0,060$ \\
\hline & 0,058 & 0,058 & 0,054 & 0,054 & 0,054 & 0,049 \\
\hline \multicolumn{7}{|l|}{ weitere Merkmale } \\
\hline \multirow{2}{*}{\multicolumn{2}{|c|}{ Geschlecht: weiblich (Ref. männlich) }} & 0,026 & 0,009 & 0,009 & 0,010 & $-0,043+$ \\
\hline & & 0,029 & 0,027 & 0,027 & 0,027 & 0,025 \\
\hline \multirow{2}{*}{\multicolumn{2}{|c|}{ Migrationshintergrund: ja (Ref.: nein) }} & $-0,038$ & $-0,006$ & $-0,006$ & $-0,009$ & $-0,007$ \\
\hline & & 0,037 & 0,045 & 0,045 & 0,045 & 0,040 \\
\hline \multirow[t]{2}{*}{ Alter der Mutter bei Geburt (in J.) } & & $0,011^{* * *}$ & 0,006 * & 0,006 * & 0,006 * & 0,004 \\
\hline & & 0,003 & 0,003 & 0,003 & 0,003 & 0,003 \\
\hline \multirow[t]{2}{*}{ Haushaltsgröße (Beginn Sek.) } & & $-0,014$ & $-0,011$ & $-0,011$ & $-0,010$ & $-0,002$ \\
\hline & & 0,022 & 0,020 & 0,020 & 0,020 & 0,018 \\
\hline \multirow[t]{2}{*}{ Haushaltsgröße (Ende Sek.) } & & $-0,002$ & $-0,011$ & $-0,010$ & $-0,009$ & 0,006 \\
\hline & & 0,021 & 0,020 & 0,020 & 0,020 & 0,018 \\
\hline \multicolumn{7}{|l|}{ Umzüge: ja (Ref. nein) } \\
\hline \multirow[t]{2}{*}{ während Grundschulzeit } & & $-0,028$ & $-0,052$ & $-0,052$ & $-0,048$ & $-0,055+$ \\
\hline & & 0,034 & 0,032 & 0,032 & 0,032 & 0,029 \\
\hline \multirow[t]{2}{*}{ während Sekundarschulzeit } & & 0,026 & 0,002 & 0,001 & 0,000 & $-0,020$ \\
\hline & & 0,040 & 0,038 & 0,038 & 0,038 & 0,034 \\
\hline höchster Bildungsabschluss der Eltern & (Ref. kein/h & ptschulabsc & uss) & & & \\
\hline Hauptschulabschl.+Ausb. & & & $-0,046$ & $-0,045$ & $-0,045$ & $-0,062$ \\
\hline & & & 0,070 & 0,070 & 0,070 & 0,062 \\
\hline mittl. Abschl.+Ausb. & & & 0,065 & 0,063 & 0,064 & $-0,063$ \\
\hline & & & 0,077 & 0,077 & 0,077 & 0,070 \\
\hline (Fach)-Hochschulreife+Ausb. & & & 0,118 & 0,117 & 0,122 & $-0,098$ \\
\hline & & & 0,097 & 0,097 & 0,097 & 0,088 \\
\hline Fachhochschule & & & $0,182+$ & $0,178+$ & $0,186+$ & 0,039 \\
\hline & & & 0,100 & 0,101 & 0,101 & 0,091 \\
\hline Universität & & & $0,439 * * *$ & $0,433 * * *$ & $0,435 * * *$ & 0,169 * \\
\hline & & & 0,091 & 0,092 & 0,091 & 0,084 \\
\hline Sonstige & & & $0,175^{* *}$ & $0,175^{* *}$ & $0,178^{* *}$ & 0,058 \\
\hline & & & 0,065 & 0,065 & 0,065 & 0,059 \\
\hline
\end{tabular}


Fortsetzung von Tabelle A2

\begin{tabular}{|c|c|c|c|c|c|c|}
\hline \multicolumn{7}{|l|}{ sozio-ökonomischer Status } \\
\hline \multirow[t]{2}{*}{ ISEI } & & & $0,004 * *$ & 0,003 * & $0,003+$ & 0,002 \\
\hline & & & 0,001 & 0,002 & 0,002 & 0,001 \\
\hline \multirow{2}{*}{\multicolumn{3}{|c|}{ relative Einkommensposition }} & & 0,025 & 0,021 & $-0,042$ \\
\hline & & & & 0,046 & 0,046 & 0,041 \\
\hline \multicolumn{7}{|c|}{ Wirtschaftliche Sorgen der Eltern (Ref.: keine Sorgen) } \\
\hline \multirow{2}{*}{ einige (Beginn Sek.) } & & & & & $-0,027$ & 0,000 \\
\hline & & & & & 0,046 & 0,041 \\
\hline \multirow[t]{2}{*}{ grosse (Beginn Sek.) } & & & & & $-0,011$ & 0,019 \\
\hline & & & & & 0,037 & 0,034 \\
\hline \multirow[t]{2}{*}{ einige (Ende Sek.) } & & & & & $-0,060$ & $-0,048$ \\
\hline & & & & & 0,050 & 0,045 \\
\hline \multicolumn{3}{|l|}{ grosse (Ende Sek.) } & & & $-0,075+$ & $-0,075+$ \\
\hline \multicolumn{7}{|c|}{ Bildungsbeteiligung (Ref: kein Hochschulbesuch bzw. andere Schulform) } \\
\hline \multirow[t]{2}{*}{ Hauptschule (Beginn Sek.) } & & & & & & $-0,017$ \\
\hline & & & & & & 0,035 \\
\hline \multirow[t]{2}{*}{ Gymnasium (Beginn Sek.) } & & & & & & $0,077+$ \\
\hline & & & & & & 0,040 \\
\hline \multirow[t]{2}{*}{ Hauptschule (Ende Sek.) } & & & & & & $-0,127 * * *$ \\
\hline & & & & & & 0,038 \\
\hline \multirow[t]{2}{*}{ Gymnasium (Ende Sek.) } & & & & & & $0,348 * * *$ \\
\hline & & & & & & 0,037 \\
\hline \multirow[t]{2}{*}{ Konstante } & $0,364^{* * *}$ & 0,177 & 0,109 & 0,097 & 0,184 & 0,236 \\
\hline & 0,020 & 0,150 & 0,164 & 0,166 & 0,173 & 0,156 \\
\hline $\mathrm{s}_{\mathrm{e}}^{2}$ & 0,362 & 0,361 & 0,355 & 0,355 & 0,357 & 0,330 \\
\hline $\mathrm{s}_{\mathrm{u}}{ }^{2}$ & 0,296 & 0,280 & 0,236 & 0,236 & 0,231 & 0,197 \\
\hline $\mathrm{N}$ & 963 & 963 & 963 & 963 & 963 & 963 \\
\hline
\end{tabular}

Anmerkungen: SOEP v31.1 (ungewichtet), random effects Modelle berücksichtigen die Abhängigkeit von Kindern mit derselben Mutter, Koeffizienten und Standardfehler (kursiv), Signifikanzniveaus: $\left.\left.\left.{ }^{* * *}\right) 0,001,{ }^{* *}\right) 0,01,{ }^{*}\right) 0,05,+$ ) 0,1 . 